\title{
THE FAILURE RATE IN RELIABILITY: APPROXIMATIONS AND BOUNDS ${ }^{1}$
}

\author{
CHRISTIANE COCOZZA-THIVENT \\ Université de Marne-la-Vallée, Equipe d'Analyse et de Mathématiques Appliquées, \\ 2 rue de la Butte Verte, 93166 Noisy-le-Grande Cedex, France \\ VLADIMIR KALASHNIKOV \\ Institute of Systems Analysis, 9, Prospect 60 let Oktyabrya \\ 117312 Moscow, Russia
}

(Received April, 1996; Revised October, 1996)

\begin{abstract}
We consider models typical to the area of reliability, and a failure rate function for processes describing the dynamics of these models. Various approximations of the failure rate function are proposed and their accuracies are investigated. The basic case studied in the paper is a regenerative model. Some interesting particular cases (Markov, semi-Markov, etc.) are considered. All proposed estimates are stated in a tractable analytic form.
\end{abstract}

This article is dedicated to the memory of Roland L. Dobrushin.

Key words: Regenerative Process, Markov Model, Redundant System, Failure Rate, Accuracy Estimate.

AMS (MOS) subject classifications: $60 \mathrm{~K} 10,60 \mathrm{~K} 15,60 \mathrm{~K} 20$.

\section{Introduction}

Like many other great scientists, R.L. Dobrushin had a knack for getting to the root of a problem due to his belief that only a simple and clear idea provides a foundation for studying any phenomena. His fantastic mathematical technique allowed him to convert such ideas into brilliant solutions of intricate problems.

This paper contains an example of how a general idea can be transformed into a solution leading to numerical results. We took a particular problem from the area of reliability that consists of the comparison of two failure rate intensities. Such a problem can be solved directly given additional assumptions imposed on the underlying system (say, that the system is Markovian). But such a solution is unsatisfactory, in general, because the additional assumptions lead to use tools which are not the best.

Our approach can be outlined in abstract terms as follows. First, we express the desired goal function in terms of auxiliary characteristics of the underlying process (which can be estimated

\footnotetext{
${ }^{1}$ This work was partly supported by Russian Foundation of Fundamental Research (grant 9501-00023), International Science Foundation and Russian Government (grant J76100), EEC (grant INTAS-93-893), and University of Marne-La-Vallée.
} 
separately) without imposing unnecessary restrictions. For example, the difference between failure rate intensities under comparison (a goal function) can be expressed in terms of passage times of various auxiliary processes (auxiliary characteristics). Second, we give estimates of auxiliary characteristics by any available method (analytical, numerical, or simulation). The choice of the method is dictated, in general, by the type of the underlying process. Note that the additional assumptions arise on the second stage only, while the expression of the goal function via auxiliary characteristics is obtained under general assumptions at the first stage. This enables us to enlarge the class of investigated systems.

The paper is concerned specifically with a so-called failure rate function that plays an important role in reliability and represents a conditional density to fail at time $t$ provided that there was no failure until $t$. It seems clear to us and is reflected in the approach that before we can establish distributional properties of random processes, we must first study their structural features (such as regeneration or Markov property) and pathwise properties.

Throughout the paper, we deal with various random processes defined on $[0, \infty)$. It is convenient to denote by $\left(\eta_{s}\right)$ a random process having state $\eta_{s}$ at time $s \in[0, \infty)$. Sometimes (when this cannot lead to a confusion) we will use a shorter notation $\eta \equiv\left(\eta_{s}\right)$. The notation $\left(\eta_{s}\right)_{0} \leq s \leq t$ refers to the restriction of $\left(\eta_{s}\right)$ to the time segment $[0, t]\left(\right.$ so, $\left.\left(\eta_{s}\right) \equiv\left(\eta_{s}\right)_{0} \leq s<\infty\right)$.

Let a random process $\left(\eta_{s}\right)$ describe the dynamics of a system and its state space $E$ be partitioned into two subsets: $E=\mathcal{H} \cup \mathscr{P}, \mathcal{H} \cap \mathscr{P}=\emptyset$. We will view $\mathcal{H}$ as a subset of "good" (or operating) states and $\mathscr{P}$ as a subset of "bad" (or failed) states assuming that $E$ is a complete separable metric space and all paths of $\left(\eta_{s}\right)$ are cadlag (that is they are right-continuous and have limits from the left). Additional restrictions on process $\left(\eta_{s}\right)$ will be imposed later on. Let

$$
\sigma=\inf \left\{s: \eta_{s} \in \mathscr{P}\right\}
$$

be the first break-down time (or lifetime) of the system. The following characteristic

$$
\lambda(t)=\lim _{\Delta \rightarrow 0} \frac{\mathbf{P}(\sigma \leq t+\Delta \mid \sigma>t)}{\Delta}
$$

is called the failure rate function for process $\left(\eta_{s}\right)$. Of course, it is nothing else but a hazard rate function for random lifetime $\sigma$. We assume that $\lambda(t)$ is defined correctly by relation (1.2). Unfortunately, function $\lambda(t)$ defined this way cannot be calculated easily, even for simple systems that are of interest in engineering. Even if we approximate lifetime $\sigma$ by another random variable (r.v.) $\sigma^{\prime}$ which is close to $\sigma$, for example in a weak topology, this does not guarantee that the hazard rate function for $\sigma^{\prime}$ is close to $\lambda(t)$ in a natural sense.

Because of this, the following characteristic, called the Vesely failure rate, is often used instead of $\lambda(t)$ :

$$
\lambda_{V}(t)=\lim _{\Delta \rightarrow 0} \frac{\mathbf{P}\left(\eta_{t+\delta} \in \mathscr{P} \mid \eta_{t} \in \mathcal{N}\right)}{\Delta}
$$

(see Cocozza-Thivent and Roussignol [4] and [5]). These two functions $\lambda(t)$ and $\lambda_{V}(t)$ have different meaning. While $\lambda(t)$ characterizes the behavior of process $\left(\eta_{s}\right)$ until its first entrance into the subset $\Phi$, function $\lambda_{V}(t)$ is concerned with the behavior of $\left(\eta_{s}\right)$ even after its first entrance into $\Phi$. Nevertheless, in many applications $\lambda_{V}(t)$ serves as an approximation of $\lambda(t)$ and it is important to give estimates of the accuracy of this approximation. In this paper, we develop a general approach for obtaining such estimates, give corresponding examples, and propose another approximation $\lambda^{0}(t)$ for $\lambda(t)$ which depends on the behavior of $\left(\eta_{s}\right)$ until its first entrance into 9 .

In all considerations, we deal with limiting (as $t \rightarrow \infty)$ characteristics, namely with $\lambda(\infty)$, $\lambda_{V}(\infty)$, and $\lambda^{0}(\infty)$, assuming that these limits exist. These characteristics are quite typical for reliability systems. Moreover, the usage of limiting characteristics instead of the prelimiting ones can be justified (see Cocozza-Thivent and Roussignol [4]). 
The paper is organized as follows. In Section 2 we consider a decomposition which is a key tool in obtaining basic results. Examples of the decomposition are given. The core of the decomposition is the representation of the dynamics of $\left(\eta_{s}\right)$ (until its first entrance to $\mathscr{P}$ ) as the dynamics of a process $\left(\eta_{s}^{0}\right)$ (obtained from $\left(\eta_{s}\right)$ by tabooing transitions to the subset $\Phi$ of failed states) subjected to failures. These failures form a Cox process (a Poisson process with an intensity $\alpha_{t}$ modulated by $\left(\eta_{s}^{0}\right)$; that is, $\alpha_{t}=\alpha\left(\left(\eta_{s}^{0}\right)_{0<s<t}\right)$ ). Such a decomposition is used in Section 3 to obtain the failure rate function $\lambda(t)$ (see (1.2)) in terms of the intensity $\alpha_{t}$. In turn, this enables us (in subsection 3.1) to write an equation determining $\lambda(\infty)$ in the case where $\left(\eta_{s}^{0}\right)$ is a regenerative process with inter-regeneration times having an exponential moment. In subsection 3.2 we propose an upper bound $\lambda^{0}$ for $\lambda(\infty)$ which is an expectation of $\alpha_{t}$ with respect to the stationary distribution of $\left(\eta_{s}^{0}\right)$ and we prove that $\lambda^{0}=\lambda_{V}(\infty)$ in particular cases (the resulting inequality $\lambda(\infty) \leq \lambda_{V}(\infty)$ is very useful in practical studies). Subsection 3.3 deals with the Vesely failure rate (1.3) and its expression in terms of stationary characteristics of the process $\left(\eta_{s}\right)$.

The remainder of the paper refers to the case where intensity $\alpha_{t}$ depends on the current state of $\left(\eta_{s}^{0}\right): \alpha_{t}=\alpha\left(\eta_{t}^{0}\right)$. In this case, $\lambda(\infty)$ can be represented as an expectation of $\alpha(\eta)$ with respect to a specific measure $\tilde{\pi}(\cdot)$. Section 4 contains a construction of a regenerative process $\tilde{\eta}$ for which $\tilde{\pi}$ is a stationary distribution. Using the mentioned constructions, we give accuracy estimates of $\lambda^{0}$ and $\lambda_{V}$ in Section 5, under different restrictions imposed on process $\left(\eta_{s}\right)$.

Various examples illuminating the estimates proposed will appear in [6] as the second part of this work. These examples are motivated by their applications in reliability area. In particular, they show how the derived estimates can be used in engineering.

\section{Decomposition of the Initial Process}

\subsection{Definitions}

Throughout the paper, we will assume that all random objects are defined on a common probability space $(\Omega, \mathcal{F}, \mathbf{P})$ with elementary outcomes $\omega \in \Omega$. But the argument $\omega$ will be skipped in most cases.

The following construction is crucial for us. Consider a random process $\left(\eta_{s}^{0}\right)$ with state space $\mathcal{M}$ and a random process $\left(M_{s}\right)$ such that

(1) for every $t$, the r.v. $M_{t}$ is a function of $\left(\eta_{s}^{0}\right)_{0} \leq s \leq t$;

(2) for every $t$, the r.v. $M_{t}$ is non-negative;

(3) the functional $M_{t}$ is a nondecreasing function of $t$.

In other words, if $\mathscr{F}_{t}^{0}$ is the $\sigma$-field generated by random variables $\eta_{s}^{0}, 0 \leq s \leq t$, and $\mathscr{F}^{0}=\left(\mathscr{F}_{t}^{0}\right)_{t>0}$ is the natural filtration generated by the history of the process $\left(\eta_{s}^{0}\right)$, then $\left(M_{t}\right)$ is an increasing process adapted to the filtration $\mathscr{F}^{0}$ (see Jacod and Shiryaev [13] (Chapter 1)). Let us call $\left(M_{t}\right)$ an integral intensity function. As we will see, the integral intensity function will be typically represented as the integral

$$
M_{t}=\int_{0}^{t} \alpha_{u} d u
$$

with respect to the Lebesgue measure, and the intensity function $\alpha_{u}=\alpha_{u}\left(\left(\eta_{s}^{0}\right)_{0<s<u}\right)$ is a nonnegative and right-continuous process, adapted to the filtration $\mathcal{F}^{0}$. To avoid pathological cases, let us assume in this paper that representation (2.1) holds true. In fact, all results below can be reformulated in terms of $M_{t}$ only, and therefore the requirement (2.1) can be relaxed.

Definition 2.1: Say that $\left(\left(\eta_{s}, \sigma\right)\right.$ can be decomposed into $\left(\left(\eta_{s}^{0}\right),\left(\alpha_{s}\right)\right)$ if

$$
\mathbf{P}\left(\left(\eta_{s}\right)_{0} \leq s \leq t \in \cdot, \sigma>t\right) \equiv \mathbf{E} I\left(\left(\eta_{s}\right)_{0 \leq s \leq t} \in \cdot, \sigma>t\right)
$$


for any $t \geq 0$.

$$
=\mathbf{E}\left(I\left(\left(\eta_{s}^{0}\right)_{0 \leq s \leq t} \in \cdot\right) \exp \left(-\int_{0}^{t} \alpha_{u} d u\right)\right)
$$

To clarify equation (2.2), let us define an r.v. $T$ jointly with $\left(\eta_{s}^{0}\right)$ by the conditional distribution

$$
\mathbf{P}\left(T>t \mid\left(\eta_{s}^{0}\right)\right)=\exp \left(-M_{t}\right)=\exp \left(-\int_{0}^{t} \alpha_{u} d u\right)
$$

It follows from the restrictions imposed on $\alpha_{u}$ that, for any $t \geq 0$,

$$
\mathbf{P}\left(T>t \mid\left(\eta_{s}^{0}\right)\right)=\mathbf{P}\left(T>t \mid\left(\eta_{s}^{0}\right)_{0} \leq s \leq t\right) \equiv \mathbf{P}\left(T>t \mid \mathcal{F}_{t}^{0}\right)
$$

Thus $\alpha_{t}$ can be viewed as a random conditional failure rate of r.v. $T$ at time $t$ given $\left(\eta_{s}^{0}\right)_{0} \leq s \leq t$. Equation (2.2) is equivalent to

$$
\mathbf{P}\left(\left(\eta_{s}\right)_{0} \leq s \leq t \in \cdot, \sigma>t\right)=\mathbf{P}\left(\left(\eta_{s}^{0}\right)_{0} \leq s \leq t \in \cdot, T>t\right) .
$$

This last equation will play a role similar to the coupling used in Cocozza-Thivent and Roussignol [4].

The following lemma is an obvious but useful consequence of Definition 2.1.

Lemma 2.2: Suppose that $\left(\left(\eta_{t}\right), \sigma\right)$ can be decomposed into $\left(\left(\eta_{t}^{0}\right),\left(\alpha_{t}\right)\right)$ and that

Then,

$$
0 \leq \alpha_{t} \leq \epsilon, \quad \forall t \geq 0
$$

$$
\mathbf{P}(\sigma>t) \geq e^{-\epsilon t}, \quad \forall t \geq 0 .
$$

This lemma means that $\sigma$ is dominated stochastically by an r.v. having an exponential law with parameter $\epsilon$ if the intensity function is bounded by $\epsilon$. The notion of stochastic domination can be found in Shaked and Shanthikumar [21] (Section 1.A) or Lindvall [18] (Chapter IV.1).

\subsection{Examples}

In this subsection we treat a variety of models partly motivated by their applications to reliability. They illustrate Definition 2.1 and contain corresponding decompositions which will be used in the sequel.

Example 2.3: Markov process on a discrete state space. Consider a time-homogeneous Markov process $\left(\eta_{s}\right)$ on a discrete state space $E$ with transition rates

$$
A(i, j)=\lim _{\Delta \rightarrow 0} \frac{\mathbf{P}\left(\eta_{t+\Delta}=j \mid \eta_{t}=i\right)}{\Delta}, i \neq j, i, j \in E .
$$

Let $\left(\eta_{s}^{0}\right)$ be a Markov process with the same initial distribution as $\left(\eta_{s}\right)$ and transition rates

$$
A^{0}(i, j)=A(i, j), i \neq j, \quad i, j \in \mathcal{M} \text {. }
$$

Define intensity $\alpha_{t}$ as

where

$$
\alpha_{t}=A\left(\eta_{t}^{0}, \mathscr{P}\right)
$$

$$
A(i, \mathscr{P})=\sum_{j \in \mathscr{P}} A(i, j)
$$

Then decomposition (2.2) holds true; the proof will be given later for a more general case (see Examples 2.4 and 2.9). This decomposition is widely used in the theory of Markov processes, and in that case Lemma 2.2 is quite intuitive. 
Example 2.4: Semi-Markov process on a discrete state space. Let $(X, T)=\left(X_{n}, T_{n}\right)_{n>0}$ be a Markov renewal process on a discrete state space $E$ having a semi-Markov kernel with density $q$, that is

$$
\mathbf{P}\left(X_{n+1}=j, T_{n+1}-T_{n} \leq t \mid X_{n}=i\right)=\int_{0}^{t} q(i, j, x) d x .
$$

Denote by $\left(\eta_{s}\right)$ the semi-Markov process associated with $(X, T)$ and defined by the equality

$$
\eta_{t}=X_{n} \text { if } T_{n} \leq t<T_{n+1} .
$$

The conditional density of $T_{1}$, given $X_{0}=i$, has the form

and its hazard rate function is equal to

$$
f(i, t)=\sum_{j \in E} q(i, j, t)
$$

$$
h(i, t)=\lim _{\Delta \rightarrow 0} \frac{1}{\Delta} \mathbf{P}\left(T_{1} \leq t+\Delta \mid X_{0}=i, T_{1}>t\right)=\frac{f(i, t)}{\int_{t}^{\infty} f(i, u) d u} .
$$

Let

$$
p(i, j, t)=\frac{q(i, j, t)}{f(i, t)}=\mathbf{P}\left(X_{1}=j \mid X_{0}=i, T_{1}=t\right) .
$$

Then the density of the semi-Markov kernel can be represented as

So the transition rate function,

$$
q(i, j, t)=p(i, j, t) f(i, t)=p(i, j, t) h(i, t) \exp \left(-\int_{0}^{t} h(i, s) d s\right) .
$$

$$
A(i, j, t)=\lim _{\Delta \rightarrow 0} \frac{1}{\Delta} \mathbf{P}\left(X_{1}=j, T_{1} \leq t+\Delta \mid X_{0}=i, T_{1}>t\right),
$$

can be expressed as

$$
A(i, j, t)=p(i, j, t) h(i, t)
$$

Since $\sum_{j \in E} p(i, j, t)=1$

and therefore,

$$
h(i, t)=\sum_{j \in E} A(i, j, t)
$$

$$
q(i, j, t)=A(i, j, t) \exp \left(-\int_{0}^{t} \sum_{k \in E} A(i, k, s) d s\right) .
$$

It follows from relation (2.10) that a semi-Markov process can be defined either by its kernel or by transition rate function.

The decomposition of a semi-Markov process is given in the following proposition.

Proposition 2.5: Let $\left(\eta_{s}\right)$ be a semi-Markov process with a finite state space $E$ and transition rate function $A(i, j, x)$ and suppose $\eta_{0} \in \mathcal{M}$. For $\mathscr{\rho} \subset E$ define

$$
A(i, \Im, t)=\sum_{j \in \Phi} A(i, j, t)
$$

and $\sigma=\inf \left\{t: \eta_{t} \in \Phi\right\}$. Let $\left(\eta_{s}^{0}\right)$ be a semi-Markov process with state space $\mathcal{M}$, the same initial distribution as $\left(\eta_{s}\right)$ and transition rates

$$
A_{0}(i, j, t)=A(i, j, t), i \neq j, i, j \in \mathcal{M} \text {. }
$$

Define the intensity function $\alpha_{t}$ by the equality

$$
\alpha_{t}=A\left(\eta_{t}^{0}, \Phi, y_{t}^{0}\right)
$$


where $y_{t}^{0}$ is the time spent by process $\left(\eta_{s}^{0}\right)$ in its current state.

Then $\left(\left(\eta_{t}\right), \sigma\right)$ can be decomposed into $\left(\left(\eta_{t}^{0}\right),\left(\alpha_{t}\right)\right)$.

Sketch of the Proof: It is sufficient to show that, for any $0<t_{1}<\ldots<t_{k}<t_{k+1}=t$ and $i_{1}, \ldots, i_{k}$ in $\mathcal{M}$

$$
\mathbf{P}\left(\eta_{t_{1}}=i_{1}, \ldots, \eta_{t_{k}}=i_{k}, \sigma>t\right)=\mathbf{E}\left(I\left(\eta_{t_{1}}^{0}=i_{1}, \ldots, \eta_{t_{k}}^{0}=i_{k}\right) \exp \left(-\int_{0}^{t} \alpha_{u} d u\right)\right)
$$

Introducing the Markov renewal process $(X, T)$ and conditioning by $\eta_{0}=X_{0}=i_{0}$, we obtain

$$
\begin{gathered}
\mathbf{P}\left(\eta_{t_{1}}=i_{1}, \ldots, \eta_{t_{k}}=i_{k}, \sigma>t \mid \eta_{0}=i_{0}\right) \\
=\sum_{0 \leq n_{1} \leq \ldots \leq n_{k} \leq n} \mathbf{P}\left(\bigcap_{j=1}^{k}\left\{X_{n_{j}}=i_{j}, T_{n_{j}} \leq t_{j}<T_{n_{j}+1}\right\}, T_{n} \leq t<T_{n+1}, \sigma>t \mid X_{0}=i_{0}\right)= \\
\sum_{N} \mathbf{P}\left(\bigcap_{r=0}^{k}\left(\left\{X_{n_{r+1}}=i_{r+1}, T_{n_{r+1}} \leq t_{r+1}<T_{n_{r+1}+1}\right\} \bigcap_{l=1}^{n_{r+1}-n-1}\left\{X_{n_{r}+l} \in \mathcal{M}\right\}\right) \mid X_{0}=i_{0}\right) \\
=\sum_{N} \sum_{r=0}^{k} \sum_{l=1}^{n_{r+1}-n_{r}-1} \sum_{x_{r+1, l} \in \mathcal{M}} \int \ldots \int\left(\prod_{r=0}^{k} q\left(i_{r}, x_{r+1,1}, u_{n_{r}+1}\right)\right. \\
\left.\quad \times \prod_{l=1}^{n_{r+1}-n_{r}-1} q\left(x_{r+1, l}, x_{r+1, l+1}, u_{n_{r}+l+1}\right)\right) \int_{t-u_{n}}^{\infty} f\left(x_{k+1, n-n_{k}-1}, s\right) d s \\
\times\left(\bigcap_{j=1}^{k}\left\{u_{1}+\ldots+u_{n_{j}} \leq t_{j}<u_{1}+\ldots+u_{n_{j}+1}\right\}, u_{1}+\ldots+u_{n} \leq t\right) d u_{1} \ldots d u_{n},
\end{gathered}
$$

where the outer $\operatorname{sum} \sum_{N}$ is taken over the set $N=\left\{\left(n_{0}, \ldots, n_{k+1}: 0=n_{0} \leq n_{1} \leq \ldots \leq n_{k} \leq\right.\right.$ $\left.n_{k+1}=n\right\}$. Let $q_{0}$ be the Markov renewal kernel density of process $\left(\eta_{s}^{0}\right)$. Equation (2.10) and a similar equation written for process $\left(\eta_{s}^{0}\right)$ show that

$$
q(i, j, u)=q_{0}(i, j, u) \exp \left(-\int_{0}^{u} A(i, \Im, s) d s\right)
$$

If $h_{0}$ is the hazard rate of the first jump time for process $\left(\eta_{s}^{0}\right)$, then

$$
\int_{u}^{+\infty} f(i, s) d s=\exp \left(-\int_{0}^{u} h(i, s) d s\right)=\exp \left(-\int_{0}^{u}\left(h_{0}(i, s)+A(i, \Im, s)\right) d s\right) .
$$

Substituting (2.14) and (2.15) into (2.13) we arrive at the desired formula (2.12).

Example 2.6: Redundant system. Let a system consist of $N$ elements. Each element can be in one of the two states 0 or 1 . State 0 corresponds to the failure of an element while state 1 means that the element is operating. Assume that all elements are independent and let $\eta_{t}=\left(\eta_{t}(1), \ldots, \eta_{t}(N)\right)$ be the state vector of the system. Assume additionally that each marginal process $\eta_{t}(i), 1 \leq i \leq N$, is an alternating renewal process. Then successive sojourn times of $\eta_{t}(i)$ at states 0 and 1 are independent r.v.'s. Denote by $\lambda^{(i)}(t)$ and $\mu^{(i)}(t)$ failure and repair rates of element $i(1 \leq i \leq N)$, respectively. In these terms, the density function of a sojourn time at state 1 (at state 0 , resp.) for element $i$ can be represented in the form

$$
f_{i}(t)=\lambda^{(i)}(t) \exp \left(-\int_{0}^{t} \lambda^{(i)}(s) d s\right)
$$


Let

$$
\text { resp. } g_{i}(t)=\mu^{(i)}(t) \exp \left(-\int_{0}^{t} \mu^{(i)}(s) d s\right)
$$

$$
y_{t}=\left(y_{t}(1), \ldots, y_{t}(N)\right)
$$

be the vector of elapsed time of elements in their current states. For instance, $y_{t}(i)$ is the time elapsed from the last (before $t$ ) jump of $\left(\eta_{s}(i)\right)$. In fact, $y_{t}$ can be viewed as a functional of $\left(\eta_{s}\right)_{0 \leq s \leq t}$. Process $\left(\eta_{s}\right)$ can be regarded as a piecewise-constant process with jumps defined by the rates

and

$$
\lambda^{(i)}\left(y_{t}(i)\right)=\lim _{\Delta \rightarrow 0} \frac{1}{\Delta} \mathbf{P}\left(\eta_{t+\Delta}(j)=\eta_{t}(j), \forall j \neq i ; \eta_{t+\Delta}(i)=0 \mid\left(\eta_{s}\right)_{0 \leq s \leq t}, \eta_{t}(i)=1\right)
$$

$$
\mu^{(i)}\left(y_{t}(i)\right)=\lim _{\Delta \rightarrow 0} \frac{1}{\Delta} \mathbf{P}\left(\eta_{t+\Delta}(j)=\eta_{t}(j), \forall j \neq i ; \eta_{t+\Delta}(i)=1 \mid\left(\eta_{s}\right)_{0 \leq s \leq t}, \eta_{t}(i)=0\right) .
$$

Now, let us assume that process $\left(\eta_{s}\right)$ can reach set $\mathscr{P}$ from $\mathcal{H}$ only at times when one of the element fails. Let $\eta=(\eta(1), \ldots, \eta(N))$ be a vector of binary components denoting a state of the system and let

$$
R_{1}(\eta)=\{k: \eta(k)=1,1 \leq k \leq N\}
$$

be the set of operating elements. For $\eta \in \mathcal{N}$, define another subset of elements (that may be empty)

such that

$$
R(\eta) \subset R_{1}(\eta)
$$

$$
k \in R(\eta) \Leftrightarrow \eta(k)=1,(\eta(1), \ldots, \eta(k-1), 0, \eta(k+1), \ldots, \eta(N)) \in \mathscr{P} .
$$

In other words, $R(\eta)$ is the set of "critical components" with respect to state $\eta$. Define a piecewise-constant process $\left(\eta_{s}^{0}\right)$ with state space $\mathcal{M}$ by the rates

$$
\lambda^{(i)}\left(y_{t}^{0}(i)\right)=\lim _{\Delta \rightarrow 0} \frac{1}{\Delta} \mathbf{P}\left(\eta_{t+\Delta}^{0}(j)=\eta_{t}^{0}(j), \forall j \neq i ; \eta_{t+\Delta}^{0}(i)=0 \mid\left(\eta_{s}^{0}\right)_{0 \leq s \leq t}, \eta_{t}^{0}(i)=1\right)
$$

and

$$
\mu^{(i)}\left(y_{t}^{0}(i)\right)=\lim _{\Delta \rightarrow 0} \frac{1}{\Delta} \mathbf{P}\left(\eta_{t+\Delta}^{0}(j)=\eta_{t}^{0}(j), \forall j \neq i ; \eta_{t+\Delta}^{0}(i)=1 \mid\left(\eta_{s}^{0}\right)_{0 \leq s \leq t}, \eta_{t}^{0}(i)=0\right),
$$

where vector $y_{t}^{0}$ of elapsed times for $\left(\eta_{s}^{0}\right)$ is defined quite similarly as $y_{t}$. Let

Then condition (2.2) is met.

$$
\alpha_{s}=\sum_{i \in R\left(\eta_{s}^{0}\right)} \lambda^{(i)}\left(y_{s}^{0}(i)\right)
$$

The proof of this result can be given by similar arguments as in the previous example. We will exhibit another approach in the following subsection and give an alternative proof for this result (see Example 2.11).

Observe that, in reliability theory, one often considers decomposed processes, where $\left(\eta_{t}^{0}\right)$ describes the system dynamics without failuring factors. The failure occurs within $[t, t+\Delta)$ with probability $\alpha_{t} \Delta$ and the intensity $\alpha_{t}$ to fail depends either on state $\eta_{t}^{0}$ or even on prehistory $\left(\eta_{s}^{0}\right)_{0} \leq s \leq t$

\subsection{A general method}

We now present a general approach that can be applied for time-homogeneous Markov processes $\left(\eta_{s}\right)$. Some aspects that are intuitively reasonable are taken at face value, in order that the basic idea may be exposed without the burden of too much mathematical detail. The assumption that $\left(\eta_{s}\right)$ is a Markov process is not restrictive since a non-Markov process can be embedded into a Markov process by equipping it with supplementary coordinates (see Example 2.11 and 
Kalashinikov [15] (Chapter 10)). Process $\left(\eta_{s}\right)$ can be defined in terms of its transition probability

$$
Q(\eta ; t, \cdot)=\mathbf{P}\left(\eta_{s+t} \in \cdot \mid \eta_{s}=\eta\right)
$$

However, this characteristic is not convenient for practical purposes since it cannot be found in a closed form for many processes of interest. In practice, one prefers to define Markov processes with the help of so-called infinitesimal characteristics. One of the most useful infinitesimal characteristics is the generator A. A naive definition of the generator can be given as follows (see Dynkin [9] (Chapters 1 and 2), Feller [11] (Chapter X), and Ethier and Kurtz [10] (Chapter 4) for rigorous definitions). Let us view the transition probability (2.19) as an operator that maps the set of measurable bounded functions $f: E \rightarrow \mathbf{R}^{1}$ into itself by the formula

$$
g_{t}(\eta)=\int_{E} f(\xi) Q(\eta ; t, d \xi)=\mathbf{E}\left(f\left(\eta_{t}\right) \mid \eta_{0}=\eta\right), \quad t \geq 0
$$

where $g_{0}(\eta)=f(\eta)$. The following characteristic is called the infinitesimal operator or generator of $\left(\eta_{s}\right)$ :

$$
g(\eta) \equiv \mathbf{A} f(\eta)=\lim _{t \rightarrow 0} \frac{g_{t}(\eta)-g_{0}(\eta)}{t}
$$

provided the limit exists in the sup-normed topology $\|f\|=\sup _{\eta}|f(\eta)| ; \mathbf{A} f(\eta)$ is a derivative of the mean value of $f\left(\eta_{t}\right)$ at $t=0$, along the path of process $\left(\eta_{s}\right)$ given $\eta_{0}=\eta$; it is not defined on the set of all measurable bounded functions (in general) but only on a subset $D_{\mathbf{A}}$ called the generator domain. This subset depends on the type of process under consideration. Actually, there are several definitions of the generator owing to the variety of meanings of the limit in (2.20) (see Dynkin [9]) and due to the expansion of the domain of $\mathbf{A}$ to unbounded functions (see Davis [7] and Kalashnikov [14]).

We restrict ourselves with only Feller processes (see Dynkin [9] (Chapter 2) and Meyn and Tweedie [19]). This class of processes can be characterized by the property that transition probabilities (2.19) map the set of bounded continuous functions into itself. It follows, in particular, that Feller processes have transition functions that are continuous with respect to the first argument (initial state).

Operator $\mathbf{A}$ is linear and there is one-to-one correspondence between $\mathbf{A}$ and transition probability $Q$ under wide conditions (see Dynkin [9] (Chapter 1)). Here, we will assume that such a correspondence takes place. Formula (2.20) is equivalent to the equation

$$
\mathbf{E}\left(f\left(\eta_{h}\right) \mid \eta_{0}=\eta\right)=f(\eta)+h \mathbf{A} f(\eta)+o(h) .
$$

The following theorem gives a general way of decomposing a Markov process.

Theorem 2.7: Let $\left(\eta_{s}\right)$ be a Markov process with state space $E$ and generator $\mathbf{A}$, and let $\mathcal{M}$ be a subset of $E, \mathscr{P}=\mathcal{H}^{c}$ and $\sigma=\inf \left\{t: \eta_{t} \in \mathscr{P}\right\}$. Suppose that $\mathbf{A}$ can be decomposed into the sum

$$
\mathbf{A}=\mathbf{A}^{0}+\mathbf{A}^{\mathfrak{\Phi}}
$$

such that $\mathbf{A}^{0} f(\eta)$ does not depend on values $f(\xi), \xi \in \mathscr{\Phi}$, if $\eta \in \mathcal{M}$, and component $\mathbf{A}^{\mathscr{P}}$ has the form

$$
\mathbf{A}^{\mathscr{P}} f(\eta)=\int_{\mathscr{P}} f(\xi) A(\eta ; d \xi)-f(\eta) A(\eta ; \mathscr{P}), \quad \eta \in E,
$$

where $A(\eta ; \cdot)$ is a finite positive measure with support $\mathscr{P}$ for each $\eta \in \mathcal{M}$, continuous with respect to $\eta \in \mathcal{M}$ and $\sup _{\eta \in \mathcal{N}} A(\eta, \mathscr{P})<\infty$. Then $\left(\left(\eta_{s}\right), \sigma\right)$ can be decomposed into $\left(\left(\eta_{s}^{0}\right),\left(\alpha_{s}\right)\right)$, where $\left(\eta_{s}^{0}\right)$ is a Markov process with generator $\mathbf{A}^{0}$ (restricted to $\left.\mathcal{M}\right)$ and

$$
\alpha_{s}=A\left(\eta_{s}^{0} ; \mathscr{\Phi}\right) \text {. }
$$

Proof: Formula (2.24) can be regarded as a consequence of more general results contained in 
Dynkin [9] (Theorem 9.7 and Sections 10.1 to 10.3) if one treats $\left(\eta_{s}\right)$ as a Markov process that exits a time $\sigma$. Our purpose here is to present an intuitive reasoning of the result rather than its rigorous version given in the cited book.

Let us estimate the probability

$$
p_{t}(B) \equiv \mathbf{P}\left(\left(\eta_{s}\right)_{0} \leq s \leq t \in B ; \sigma>t\right)
$$

for fixed time $t>0$ and subset $B$. Process $\left(\eta_{s}\right)$ has right-continuous paths. Therefore, it is sufficient to find probability $(2.25)$ for a cylindric set $B$ that is to take $B$ of the form

$$
\left\{\omega:\left(\eta_{s}\right)_{0} \leq s \leq t(\omega) \in B\right\}=\left\{\omega: \eta_{t}(\omega) \in B_{j} \subset \mathcal{N}, 1 \leq j \leq n, 0 \leq t_{1}<t_{2}<\ldots<t_{n} \leq t\right\}
$$

where $n \geq 1$ and $0 \leq t_{1}<t_{2}<\ldots<t_{n} \leq t$ are arbitrary.

Let us define a killed Markov process $\left(\widetilde{\eta}_{s}\right)$ (see Dynkin [9], (Chapter 10)):

$$
\tilde{\eta}_{s}=I(\sigma>s) \eta_{s}+I(\sigma \leq s) \phi .
$$

This means that the state $\widetilde{\eta}_{s}$ is not defined after stopping time $\sigma$. Denote by $\tilde{\mathbf{A}}$ the generator of $\left(\widetilde{\eta}_{s}\right)$. In these terms,

$$
p_{t}(B) \equiv \mathbf{P}\left(\tilde{\eta}_{t} \in B_{j}, 1 \leq j \leq n, \tilde{\eta}_{t} \in \mathcal{M}\right) .
$$

Let us take a function $f$ from the domain of operator $\mathbf{A}^{0}$ that is equal to zero in $\mathscr{P}$. Then, by $(2.22)$ and (2.23),

$$
\mathbf{A} f(\eta)=\tilde{\mathbf{A}} f(\eta)=\mathbf{A}^{0} f(\eta)-A(\eta, \mathscr{\Im}) f(\eta), \quad \eta \in \mathcal{M} .
$$

This means (see (2.21)) that, for any $\eta \in \mathcal{H}$,

$$
\begin{array}{r}
\mathbf{E}\left(f\left(\tilde{\eta}_{h}\right) \mid \tilde{\eta}_{0}=\eta\right)=f(\eta)+h \mathbf{A}^{0} f(\eta)-h A(\eta ; \mathscr{P}) f(\eta)+o(h) \\
=(1-A(\eta ; \mathscr{P}) h)\left(f(\eta)+h \mathbf{A}^{0} f(\eta)\right)+o(h) \\
=e^{-A(\eta ; \mathscr{P}) h} \mathbf{E}\left(f\left(\eta_{h}^{0}\right) \mid \eta_{0}^{0}=\eta\right)+o(h) \\
=\mathbf{E}\left(f\left(\eta_{h}^{0}\right) \exp \left(-\int_{0}^{h} A\left(\eta_{s}^{0} ; \mathscr{P}\right) d s\right) \mid \eta_{0}^{0}=\eta\right)+o(h) .
\end{array}
$$

As there is one-to-one correspondence between transition functions and their generators, we have, for any $C \subset \mathcal{M}$,

$$
\mathbf{P}\left(\tilde{\eta}_{h} \in C \mid \tilde{\eta}_{0}=\eta\right)=\mathbf{E}\left(I\left(\eta_{h}^{0} \in C\right) \exp \left(-\int_{0}^{h} A\left(\eta_{u}^{0} ; \mathfrak{P}\right) d u\right) \mid \eta_{0}^{0}=\eta\right)+o(h) .
$$

Using the Markov property of processes $\left(\widetilde{\eta}_{s}\right)$ and $\left(\eta_{s}^{0}\right)$ and equality $(2.31)$, and letting $h \rightarrow 0$, we have for any $s=m h, m \geq 1$,

$$
\begin{gathered}
\mathbf{P}\left(\tilde{\eta}_{s} \in C\right)=\lim _{m \rightarrow \infty} \mathbf{E}\left(\prod_{j=0}^{m-2} \mathbf{P}\left(\tilde{\eta}_{(j+1) h} \in \mathcal{M} \mid \widetilde{\eta}_{j}\right) \mathbf{P}\left(\tilde{\eta}_{m h} \in C \mid \widetilde{\eta}_{s-h}\right)\right) \\
=\lim _{m \rightarrow \infty} \mathbf{E}\left(\mathbf{P}\left(\eta_{s}^{0} \in C \mid \eta_{s-h}^{0}\right) \mathbf{E} \prod_{j=0}^{m-2} \exp \left(-\int_{j h}^{(j+1) h} A\left(\eta_{u}^{0} ; \mathscr{P}\right) d u\right)\right) \\
=\mathbf{E}\left(I\left(\eta_{s}^{0} \in C\right) \exp \left(-\int_{0}^{s} A\left(\eta_{u}^{0} ; \mathscr{P}\right) d u\right)\right) .
\end{gathered}
$$

This and the Markov property imply that the probability (2.28) has the form 
This yields the result.

$$
p_{t}(B)=\mathbf{E}\left(I\left(\eta_{t_{j}}^{0} \in B_{j}, 1 \leq j \leq n\right) \exp \left(-\int_{0}^{t} A\left(\eta_{u}^{0} ; \mathscr{P}\right) d u\right)\right)
$$

Remark 2.8: It is useful to know that $\mathbf{1}_{\eta \in \mathcal{M}} \in D_{\mathbf{A}}$ and therefore $\mathbf{1}_{\eta \in \mathscr{P}}=1-\mathbf{1}_{\eta \in \mathcal{N}} \in D_{\mathbf{A}}$. To prove this, note that $f(\eta) \equiv 1, \eta \in \mathcal{M}$, belongs to $D_{\mathbf{A}^{0}}$. This function $f$ definitely belongs to the domain $D_{\overline{\mathbf{A}}}$ (where $\overline{\mathbf{A}}$ is defined in (2.29)) by (2.20), (2.21), and (2.22). In fact, $\tilde{\mathbf{A}}$ is the generator of the killed Markov process that exits at time $\sigma$ and $D_{\overline{\mathbf{A}}}=D_{\mathbf{A}^{0}}$ by Theorem 9.3 from Dynkin [9]. Let us extend $f$ as follows:

$$
f_{\text {gP }}(\eta)=\left\{\begin{array}{l}
1, \text { if } \eta \in \mathcal{M} \\
0, \text { if } \eta \in \mathscr{P}
\end{array}\right.
$$

Such an extended function belongs to $D_{\mathbf{A}}$ just because of the definition of generators $\widetilde{\mathbf{A}}, \mathbf{A}$, and equality (2.29).

Process $\left(\eta_{s}^{0}\right)$ may contain both jumpwise and continuous components (see Examples 2.10 and 2.11). But, due to form (2.23) of operator $\mathbf{A}^{\mathscr{\rho}}$, process $\left(\eta_{s}\right)$ may hit set $\mathscr{P}$ only by jumps, and the intensity of the jump at point $x \in E$ is equal to $A(x ; \mathscr{\rho})$. If such a jump leads to subset $\mathscr{P}$, then the state of process $\left(\eta_{s}\right)$ just after the jump is random with the probability distribution $A(x ; \cdot) / A(x ; \mathscr{\rho})$, while process $\left(\eta_{s}^{0}\right)$ stays in $\mathcal{M}$.

Example 2.9: Markov process on a discrete state space. Consider process $\left(\eta_{s}\right)$ from Example 2.3. By definition (2.6), its generator has the form

$$
\mathbf{A} f(i)=\sum_{j \in E} A(i, j)(f(j)-f(i)), \quad i \in E .
$$

Let us decompose the operator $\mathbf{A}$ in the following way:

$$
\mathbf{A}=\mathbf{A}^{0}+\mathbf{A}^{\mathfrak{P}}
$$

where

and

$$
\mathbf{A}^{0} f(i)=\sum_{j \in \mathcal{M}} A(i, j)(f(j)-f(i)), i \in E,
$$

$$
\mathbf{A}^{\mathscr{P}} f(i)=\sum_{j \in \mathscr{P}} A(i, j)(f(j)-f(i)), \quad i \in E .
$$

Then, all conditions of Theorem 2.7 are fulfilled and formulas (2.8) and (2.24) coincide.

Example 2.10: Semi-Markov process on a discrete state space. Consider a semi-Markov process $\left(\eta_{s}\right)$ from Example 2.4 and denote by $y_{s}$ the elapsed time of the process in its current state at time $s$. Then process $\eta_{s}^{\prime}=\left(\eta_{s}, y_{s}\right), s \geq 0$, is Markov and its generator has the form

$$
\mathbf{A} f(i, y)=\frac{\partial f(i, y)}{\partial y}+\sum_{j \in E} A(i, j, y)(f(j, 0)-f(i, y)) .
$$

Let $\mathcal{H}^{\prime}=\mathcal{M} \times[0, \infty)$ and $\mathscr{P}^{\prime}=\mathscr{P} \times[0, \infty)$. This means that $(i, y) \in \mathcal{H}^{\prime}$ if and only if $i \in \mathcal{M}$ and $(i, y) \in \mathscr{P}^{\prime}$ if and only if $i \in \mathscr{P}$. The term $\partial f(i, y) / \partial y$ in the above formula is responsible for deterministic behavior of $y_{t}$ between successive jumps of $\left(\eta_{s}\right)$, and, for $(i, y) \in \mathcal{H}^{\prime}$, it does not depend on values of $f(j, z),(j, z) \in \mathscr{P}^{\prime}$. We arrive at the decomposition

with

$$
\mathbf{A}=\mathbf{A}^{0}+\mathbf{A}^{\mathscr{P}}
$$

$$
\mathbf{A}^{0} f(i, y)=\frac{\partial f(i, y)}{\partial y}+\sum_{j \in \mathcal{M}} A(i, j, y)(f(j, 0)-f(i, y))
$$




$$
\mathbf{A}^{\mathscr{P}} f(i, y)=\sum_{j \in \mathscr{P}} A(i, j, y)(f(j, 0)-f(i, y)) .
$$

The expression for $A(\eta ; \cdot)$ given in Theorem 2.7 (see equation $(2.23)$ ), for $\eta=(i, y)$, is equal to $A(i, \cdot, y)$ and formula (2.11) agrees with (2.24).

Example 2.11: Redundant system. Consider process $\left(\eta_{s}\right)$ from Example 2.6. If $\eta_{s}^{\prime}=\left(\eta_{s}, y_{s}\right)$, where $y_{s}$ is defined by $(2.16)$, then $\left(\eta_{s}^{\prime}\right)$ is a Markov process. It can be verified that

$$
\mathbf{A} f(\eta, y)=\sum_{i=1}^{N} \frac{\partial f(\eta, y)}{\partial y_{i}}+\sum_{i=1}^{N} a^{(i)}(\eta, y)\left(f\left(\eta^{(i)}, y^{(i)}-f(\eta, y)\right)\right.
$$

where $\eta=(\eta(1), \ldots, \eta(N)), y=(y(1), \ldots, y(N))$,

$$
\begin{gathered}
a^{(i)}(\eta, y)=\left\{\begin{array}{lc}
\lambda^{(i)}(y(i)), & \text { if } \eta(i)=1, \\
\mu^{(i)}(y(i)), & \text { if } \eta(i)=0,
\end{array}\right. \\
\eta^{(i)}(j)=\eta(j), j \neq i ; \quad \eta^{(i)}(i)=1-\eta(i) ; \\
y^{(i)}(j)=y(j), j \neq i ; \quad y^{(i)}(i)=0 .
\end{gathered}
$$

Let $\mathcal{H}^{\prime}=\mathcal{H} \times[0, \infty)^{N}$ and $\mathscr{P}^{\prime}=\mathscr{P} \times[0, \infty)^{N}$. Decompose operator $\mathbf{A}$ in the same way as in Example 2.10

where

$$
\mathbf{A}=\mathbf{A}^{0}+\mathbf{A}^{\mathscr{\rho}}
$$

$$
\mathbf{A}^{0} f(\eta, y)=\sum_{i=1}^{N} \frac{\partial f(\eta, y)}{\partial y_{i}}+\sum_{i \notin R(\eta)} a^{(i)}(\eta, y)\left(f\left(\eta^{(i)}, y^{(i)}\right)-f(\eta, y)\right)
$$

(the set $R(\eta)$ is defined in (2.17)) and

In this case,

$$
\begin{gathered}
\mathbf{A}^{\mathscr{P}} f(\eta, y)=\sum_{i \in R(\eta)} a^{(i)}(\eta, y)\left(f\left(\eta^{(i)}, y^{(i)}\right)-f(\eta, y)\right) . \\
A((\eta, y) ; \mathscr{P})=\sum_{i \in R(\eta)} a^{(i)}(\eta, y) .
\end{gathered}
$$

Using Theorem 2.7, we arrive at formula (2.18).

\section{The Asymptotic Failure Rate and its Approximations}

\subsection{The asymptotic failure rate}

The failure rate $\lambda(t)$ and its limiting value $\lambda(\infty)$ provide useful characteristics for doing a number of calculations in reliability. In this section we obtain explicit expressions for them and their approximations. To state our results, let us assume that the intensity function $\alpha_{t}=$ $\alpha_{t}\left(\left(\eta_{s}^{0}\right)_{0 \leq s \leq t}\right)$ satisfies the following conditions:

\section{Conditions $\mathbf{C}(\alpha)$}

1. $\quad\left(\left(\eta_{s}\right), \sigma\right)$ can be decomposed into $\left(\left(\eta_{s}^{0}\right),\left(\alpha_{s}\right)\right)$.

2. For each $t \geq 0$, function $\alpha_{s} \equiv \alpha_{s}(\omega)$ is right-continuous at $s=t$ for almost all $\omega \in \Omega$.

3. There exists an absolute constant $\bar{\alpha}$ such that

$$
\sup _{s} \alpha_{s}=\bar{\alpha}<+\infty
$$


The first condition is crucial to our approach. The second condition is quite natural because of the assumption that paths of $\left(\eta_{s}^{0}\right)$ are cadlag. The third assumption is restrictive, of course. For example, it may fail for semi-Markov models when holding time have IFR distributions with unbounded intensities. Moreover, some final formulas in this work hold true in the case when conditions $\mathrm{C}(\alpha)$ are violated. This means that the third assumption can be relaxed. It seems that the most straightforward and general way to do this is to find comparison estimates which yield that the desired characteristics of the process with unbounded function $\alpha_{t}$ can be obtained as the limit of corresponding characteristics for the process with intensity $\min \left(\alpha_{t}, \bar{\alpha}\right)$ when $\bar{\alpha} \rightarrow \infty$. We will not touch this problem here but refer the reader to works of Kalashnikov [16] and [17] where such problems have been solved for specific characteristics of regenerative processes. Perhaps, comparison estimates wanted for the purposes of this paper may lead to different setups in comparison theorem.

We now express the failure rate function $\lambda(t)$ introduced in (1.2) in terms of the "decomposition components" of $\left(\left(\eta_{s}, \sigma\right)\right.$.

Lemma 3.1: Let conditions $C(\alpha)$ hold. Then

$$
\lambda(t)=\frac{\mathbf{E}\left(\alpha_{t} \exp \left(-\int_{0}^{t} \alpha_{u} d u\right)\right)}{\mathbf{E} \exp \left(-\int_{0}^{t} \alpha_{u} d u\right)} .
$$

Proof: We have, by (1.2) and (2.2),

$$
\begin{gathered}
\lambda(t)=\lim _{\Delta \rightarrow 0} \frac{1}{\Delta} \frac{\mathbf{P}(\sigma>t)-\mathbf{P}(\sigma>t+\Delta)}{\mathbf{P}(\sigma>t)} \\
=\frac{1}{\mathbf{E} \exp \left(-\int_{0}^{t} \alpha_{u} d u\right)} \lim _{\Delta \rightarrow 0} \frac{1}{\Delta} \mathbf{E}\left(\exp \left(-\int_{0}^{t} \alpha_{u} d u\right)-\exp \left(-\int_{0}^{t+\Delta} \alpha_{u} d u\right)\right) .
\end{gathered}
$$

By condition $\mathrm{C}(\alpha) .2$, for every $t$ there exists a right derivative $\frac{d}{d t} \int_{0}^{t} \alpha_{u}(\omega) d u=\alpha_{t}(\omega)$ (a.s.). As

$$
\frac{1}{\Delta}\left(\exp \left(-\int_{0}^{t} \alpha_{u} d u\right)-\exp \left(-\int_{0}^{t+\Delta} \alpha_{u} d u\right)\right) \leq \exp \left(-\int_{0}^{t} \alpha_{u} d u\right) \sup _{t \leq s \leq t+1} \alpha_{s}
$$

for any $0<\Delta \leq 1$, the Lebesgue dominated convergence theorem yields

$$
\lim _{\Delta \rightarrow 0} \frac{1}{\Delta} \mathbf{E}\left(\exp \left(-\int_{0}^{t} \alpha_{u} d u\right)-\exp \left(-\int_{0}^{t+\Delta} \alpha_{u} d u\right)\right)=\mathbf{E}\left(\alpha_{t} \exp \left(-\int_{0}^{t} \alpha_{u} d u\right)\right)
$$

This completes the proof.

Let us find sufficient conditions ensuring the existence of the limit

$$
\lambda(\infty)=\lim _{t \rightarrow \infty} \lambda(t)
$$

For this, we use a "regeneration technique" (see Kalashnikov [16]). Let us recall that a probability distribution $\nu$ is spread out if there exists an $n$ such that $\nu^{* n}$ has a non-zero absolutely continuous component (see Asmussen [1] (Chapter VI)). A random variable is said to be spread out if its probability distribution is spread out. 


\section{Conditions C0}

1. Conditions $\mathrm{C}(\alpha)$ hold true.

2. Process $\left(\eta_{s}^{0}\right)$ is regenerative (in the sense of Smith) with the sequence of regeneration times $0=S_{0}, S_{1}, S_{2}, \ldots$ Let $S=S_{1}$ be spread out, $\mathbf{E} S<\infty, N(u)=\max \left\{n: S_{n} \leq u\right\}$. Assume that process $\left(\alpha_{s}\right)$ has the "lack-of-memory property" that is $\alpha_{u}$ only depends on $\left(\eta_{s}^{0}\right)_{S_{N(u)}} \leq s \leq u$.

3. The following analog of Cramér's condition holds: there exists a real number $\kappa>0$ such that

$$
\operatorname{Eexp}\left(-\int_{0}^{S}\left(\alpha_{u}-\kappa\right) d u\right)=1
$$

4. There exists $a>\kappa$ such that $\mathbf{E}\left(e^{a S}\right)<+\infty$.

Let us discuss the above conditions. Assumption $\mathrm{C} 0.2$ that $\left(\eta_{s}^{0}\right)$ is a regenerative process is quite natural from a practical point of view. In reliability, the regeneration property is often associated with returning of the process to the state where all components are operating. In accordance with this, the requirement of the lack-of-memory property for $\left(\alpha_{s}\right)$ seems to be natural. It follows that process $\left(\alpha_{s}\right)$ is also regenerative with the same regeneration times $S_{0}, S_{1}, S_{2}, \ldots$. Hypothesis $\mathrm{C} 0.4$ requires the existence of an exponential moment for inter-regeneration time $S$. This is necessary (but not sufficient, in general) for satisfying Cramér's condition (3.2) which is widely used in various applications since it enables one to simplify studying of underlying models. As we will see, $\kappa$ is small for highly reliable systems. Therefore, assumption $a>\kappa$ in hypothesis C0.4 is not restrictive in many practical reliability problems. However, condition C0.4 does not seem quite satisfactory, even for Markov processes with a finite number of states. Because of this, we will relax it in some particular cases.

The following theorem contains the desired representation of the limiting value $\lambda(\infty)$.

Theorem 3.2: Suppose that Conditions C0 hold true. Then

$$
\lambda(\infty)=\frac{\mathbf{E} \int_{0}^{S} \alpha_{t} \exp \left(-\int_{0}^{t}\left(\alpha_{u}-\kappa\right) d u\right) d t}{\mathbf{E} \int_{0}^{S} \exp \left(-\int_{0}^{t}\left(\alpha_{u}-\kappa\right) d u\right) d t}
$$

and

$$
\lambda(\infty)=\kappa .
$$

Proof: By Lemma 3.1, the following identity holds for any real $\kappa$ :

$$
\lambda(t)=\frac{\mathbf{E}\left(\alpha_{t} \exp \left(-\int_{0}^{t}\left(\alpha_{u}-\kappa\right) d u\right)\right)}{\mathbf{E} \exp \left(-\int_{0}^{t}\left(\alpha_{u}-\kappa\right) d u\right)} \equiv \frac{N_{t}}{D_{t}}
$$

Now, take $\kappa>0$ to satisfy (3.2) and prove that there exist limits for both numerator $N_{t}$ and denominator $D_{t}$. Since $\left(\eta_{s}^{0}\right)$ is a zero-delayed regenerative process and the same is true for $\left(\alpha_{s}\right), N_{t}$ satisfies the renewal equation

$$
N_{t}=\mathbf{E}\left(\alpha_{t} \exp \left(-\int_{0}^{t}\left(\alpha_{u}-\kappa\right) d u\right), S>t\right)+\int_{0}^{t} N_{t-u} \mathbf{E}\left(\exp \left(-\int_{0}^{S}\left(\alpha_{v}-\kappa\right) d v\right) I(S \in d u)\right)
$$


Let

$$
B(d u)=\mathbf{E}\left(\exp \left(-\int_{0}^{S}\left(\alpha_{v}-\kappa\right) d v\right) I(s \in d u)\right)
$$

By $(3.2), B(\cdot)$ is a probability measure. Condition C0.4 implies that

$$
b_{1}=\int_{0}^{\infty} u B(d u)<\infty
$$

As $S$ is spread out, the distribution $B$ has the same property. By C0.3 and C0.4,

$$
g(t)=\mathbf{E}\left(\alpha_{t} \exp \left(-\int_{0}^{t}\left(\alpha_{u}-\kappa\right) d u\right), S>t\right)
$$

is bounded, Lebesgue integrable and $\lim _{t \rightarrow \infty} g(t)=0$. By the key renewal theorem (see Asmussen [1] (Chapter VI, Corollary 1.3)) the following limit exists:

Quite similarly,

$$
N \equiv \lim _{t \rightarrow \infty} N_{t}=\frac{1}{b_{1}} \mathbf{E} \int_{0}^{S} \alpha_{t} \exp \left(-\int_{0}^{t}\left(\alpha_{u}-\kappa\right) d u\right)
$$

$$
D \equiv \lim _{t \rightarrow \infty} D_{t}=\frac{1}{b_{1}} \mathbf{E} \int_{0}^{S} \exp \left(-\int_{0}^{t}\left(\alpha_{u}-\kappa\right) d u\right)
$$

Relations (3.5), (3.6), and (3.7) yield (3.3). Now, by (3.2),

$$
\mathbf{E} \int_{0}^{S} \alpha_{t} \exp \left(-\int_{0}^{t}\left(\alpha_{u}-\kappa\right) d u\right) d t=\kappa \mathbf{E} \int_{0}^{S} \exp \left(-\int_{0}^{t}\left(\alpha_{u}-\kappa\right) d u\right) d t .
$$

Substituting (3.8) into (3.3) we arrive at (3.4).

\subsection{An upper bound of $\lambda(\infty)$}

Theorem 3.2 has the following useful corollaries giving upper bounds of $\lambda(\infty)$ in terms of a stationary expectation of regenerative process $\left(\eta_{s}^{0}\right)$.

Proposition 3.3: Suppose that Conditions CO hold true. Then

$$
\lambda(\infty) \leq \lambda^{0} \equiv \frac{\mathbf{E} \int_{0}^{S} \alpha_{u} d u}{\mathbf{E} S} .
$$

Proof: Applying the Jensen inequality we have from (3.2) that

Therefore,

$$
1=\mathbf{E} \exp \left(-\int_{0}^{S}\left(\alpha_{u}-\kappa\right) d u\right) \geq \exp \left(-\mathbf{E} \int_{0}^{S}\left(\alpha_{u}-\kappa\right) d u\right)
$$

$$
\mathbf{E} \int_{0}^{S}\left(\kappa-\alpha_{u}\right) d u \leq 0
$$

Since $\mathbf{E} S<\infty$ and $\lambda(\infty)=\kappa$ we arrive at (3.9).

Note that the right-hand side of (3.9) is nothing else but the stationary expectation of states of the regenerative process $\left(\alpha_{s}\right)$. 
Remark 3.4: Inequality (3.10) holds under wider assumptions than those listed in Conditions C0. Indeed, it can be used whenever we know that Condition C0.3 holds true, $\lambda(\infty) \leq \kappa$, and $\mathbf{E} S<\infty$.

Remark 3.5: Just to illustrate Remark 3.4, suppose that $\left(\eta_{s}\right)$ is an irreducible Markov or semi-Markov process on a finite state space $E$, with, for example, initial state $0 \in E$. Recall that $\left(\eta_{s}^{0}\right)$ is supposed to be regenerative, therefore state 0 is recurrent for both processes $\left(\eta_{s}^{0}\right)$ and $\left(\eta_{s}\right)$. Then they are regenerative with the first regeneration time, respectively, $S=$ $\inf \left\{s: \eta_{s}^{0}=0, \eta_{s-}^{0} \neq 0\right\}$ and $\bar{S}=\inf \left\{s: \eta_{s}=0, \eta_{s-} \neq 0\right\}$. It is easy to verify (using the same method as in the proof of Proposition 2.5) that

$$
\mathbf{E} \exp \left(-\int_{0}^{S}\left(\alpha\left(\eta_{u}^{0}\right)-\kappa\right) d u\right)=\mathbf{E}\left(e^{\kappa \bar{S}} \mathbf{1}_{\{\bar{S}<\sigma\}}\right)
$$

and the Cramér condition (3.2) is nothing else but the $r$-recurrence property (see Cocozza-Thivent and Roussignol [3]) with $r=-\kappa$. Hence, the Cramér condition (3.2) is true if distributions of sojourn time in the states have rational Laplace transforms. Moreover, Theorem 6.3 of CocozzaThivent and Roussignol [3] asserts that $\lambda(\infty)=\kappa$ under these conditions. So all hypotheses in Proposition 3.3 are fulfilled (without the necessity to turn to Assumption C0.4).

The following remark gives a useful representation of $\lambda^{0}$ for Markov processes. It is an immediate consequence of definition (3.9) of $\lambda^{0}$.

Remark 3.6: Let $\left(\eta_{s}\right)$ be a Markov process conforming to the conditions of Theorem 2.7, $\mathrm{C} 0.1$ and $\mathrm{C} 0.2$. Then

$$
\lambda^{0}=\int A(\eta ; \Phi) \pi^{0}(d \eta)
$$

$\pi^{0}$ being the stationary distribution of Markov process $\left(\eta_{s}^{0}\right)$.

Since the behavior of process $\left(\eta_{s}^{0}\right)$ depends on the behavior of $\left(\eta_{s}\right)$ until the first hitting time of the subset $\mathscr{T}$, the estimates contained in Proposition 3.3 and its Corollary 3.6 are attractive for practical use. Intensity $\lambda^{0}$ (see (3.9) to (3.11)) has a clear interpretation and can be calculated in terms of stationary characteristics of the auxiliary process $\left(\eta_{s}^{0}\right)$. We will investigate an accuracy of this approximation in Subsection 5.1.

The following lemma shows that stationary characteristics of $\left(\eta_{s}^{0}\right)$ can be obtained in terms of stationary characteristics of process $\left(\eta_{s}\right)$ provided that $\left(\eta_{s}^{0}\right)$ is time-reversible.

Given a Markov process on a countable state space $E$ with transition rates matrix $A$ and subset $\mathcal{M} \subset E$, we say that $\mathcal{M}$ is communicative if, for any $i$ and $j$ in $\mathcal{H}$, there exists a path from $i$ to $j$, i.e., there exists $n$ and $i_{1}, \ldots, i_{n}$ in $\mathcal{M}$ such that

$$
i_{1}=i, i_{n}=j, A\left(i_{m}, i_{m+1}\right)>0 \quad(1 \leq m \leq n-1) .
$$

If $E$ is communicative, the process is called irreducible. The process is time reversible if there exists a probability distribution $\pi$ such that for any $i$ and $j$,

$$
\pi(i) A(i, j)=\pi(j) A(j, i) .
$$

It can be easily seen that every distribution $\pi$ satisfying (3.12) is stationary.

Lemma 3.7: Let us consider a time-reversible Markov process $\left(\eta_{s}\right)$ on a discrete state space with stationary probability distribution $\pi(\cdot)$, and let us suppose that set $\mathcal{H}$ is communicative. Then

$$
\pi^{0}(\eta)=\frac{\pi(\eta)}{\pi(\mathcal{M})}, \quad \eta \in \mathcal{M}
$$

Proof: Let $A$ be the transition matrix (or generator) of the Markov process $\left(\eta_{s}\right)$. The 
irreducibility and reversibility yield for any $i$ and $j$ (see Disney and Kiessler [8] (Section 2.13.2)) that

$$
\begin{gathered}
\pi(i)>0, \\
A(i, j)>0 \Leftrightarrow A(j, i)>0 .
\end{gathered}
$$

Now, let $j_{0}$ be a (fixed) element of $\mathcal{M}$ and for any $j$ in $\mathcal{N}$, we choose a path from $j_{0}$ to $j$

$$
j_{0}=i_{1}, i_{2}, \ldots, i_{n-1}, i_{n}=j
$$

and define

Equation (3.12) implies

$$
\phi(j)=\frac{A\left(j_{0}, i_{2}\right) A\left(i_{2}, i_{3}\right) \ldots A\left(i_{n-1}, j\right)}{A\left(i_{2}, j_{0}\right) A\left(i_{3}, i_{2}\right) \ldots A\left(j, i_{n-1}\right)}
$$

$$
\pi(j)=\phi(j) \pi\left(j_{0}\right)
$$

Denote by $A_{0}$ the transition matrix of process $\left(\eta_{s}^{0}\right)$. By (2.7), we have

$$
A(i, j)=A^{0}(i, j)
$$

for all $i$ and $j$ from $\mathcal{M}$. Let us now define the distribution $\pi^{0}$ on $\mathcal{M}$ by

$$
\pi^{0}(j)=\phi(j) \pi^{0}\left(j_{0}\right), j \in \mathcal{H} \text {. }
$$

Given $j$ and $k$ from $\mathcal{H}$ such that $A(j, k)>0$, we have

$$
\frac{\pi^{0}(j)}{\pi^{0}(k)}=\frac{\phi(j)}{\phi(k)}=\frac{\pi(j)}{\pi(k)}=\frac{A(k, j)}{A(j, k)}=\frac{A^{0}(k, j)}{A^{0}(j, k)}
$$

(see (3.15), (3.13), (3.12), and (3.14)). Therefore, measure $\pi^{0}$ is a stationary distribution of states of process $\left(\eta_{s}^{0}\right)$. By (3.13) and (3.15), we conclude that measures $\pi$ and $\pi^{0}$ are proportional on $\mathcal{N}$, which yields the proof.

\subsection{The Vesely failure rate}

We now derive some useful relations for the Vesely failure rate. Let us start with an ergodic Markov process $\left(\eta_{s}\right)$ with a discrete state space $E$ and generator $A$. Here "ergodic" means that the Markov process has a unique stationary probability distribution $\pi$ such that

$$
\lim _{t \rightarrow \infty} \mathbf{E}\left(f\left(\eta_{t}\right)\right)=\sum_{\eta \in E} f(\eta) \pi(\eta)
$$

for every bounded function $f$ on $E$. Any irreducible Markov process on a finite state space is ergodic. It follows from (1.3) that the Vesely failure rate has the form

$$
\lambda_{V}(t)=\frac{1}{\mathbf{P}\left(\eta_{t} \in \mathcal{M}\right)} \sum_{\eta \in \mathcal{H}} \sum_{\xi \in \mathscr{P}} A(\eta, \xi) \mathbf{P}\left(\eta_{t}=\eta\right)=\frac{1}{\mathbf{P}\left(\eta_{t} \in \mathcal{M}\right)} \mathbf{E}\left(\alpha\left(\eta_{t}\right) 1_{\left\{\eta_{t} \in \mathcal{M}\right\}}\right)
$$

and consequently, under Condition $\mathrm{C}(\alpha) .3$,

$$
\lambda_{V}(\infty)=\lim _{t \rightarrow \infty} \lambda_{V}(t)=\frac{1}{\pi(\mathcal{H})} \sum_{\eta \in E} \alpha(\eta) 1_{\{\eta \in \mathcal{M}\}} \pi(\eta)=\sum_{\eta \in \mathcal{M}} \frac{\pi(\eta)}{\pi(\mathcal{H})} A(\eta, \mathscr{P}) .
$$

Proposition 3.8: Let $\left(\eta_{s}\right)$ be a time-reversible Markov process on a finite state space with a stationary distribution of states $\pi(\cdot)$ and a bounded generator $A$; let set $\mathcal{M}$ be communicative. Then,

and

$$
\lambda_{V}(\infty)=\lambda^{0}
$$

$$
\lambda(\infty) \leq \lambda_{V}(\infty)
$$


Proof: The proof follows from Example 2.3, Remark 3.6, Lemma 3.7, and formula (3.16) by using the result indicated in Remark 3.5.

Remark 3.9: Engineers are often interested in the Vesely failure rate since it can easily be computed. Such calculations can be done with the help of a fault tree when the system consists of independent components (see Example 3.14). In such a case the system behavior can be described by a finite reversible Markov process, if the failure and repair rates of each component are constant and strictly positive. Engineers observed (from practical examples) that $\lambda(\infty) \leq \lambda_{V}(\infty)$ but, as far as we know, this result had not been proved. Now it is done and it is an important result for applications.

As we will see in Proposition 3.15, the equality $\lambda^{0}=\lambda_{V}(\infty)$ is still true for a general redundant system introduced in Example 2.6 (see also Example 3.14) in the case of constant failure rates and general repair rates.

We now come back to general Markov processes. The following proposition generalizes representation (3.16) of general Markov processes having the Feller property.

Proposition 3.10: Let $\left(\eta_{s}\right)$ be an ergodic Feller process satisfying assumptions of Theorem 2.7 with stationary distribution of states $\pi$. Then

$$
\lambda_{V}(\infty)=\frac{1}{\pi(\mathcal{M})} \int_{\mathcal{M}} A(\eta ; \Phi) \pi(d \eta)
$$

Proof: By Remark 2.8, function $f(\eta)=1_{\{\eta \in \mathscr{P}\}}$ belongs to the domain of the generator of $\left(\eta_{s}\right)$. The generator's definition and (1.3) yield

$$
\begin{gathered}
\lambda_{V}(t)=\frac{1}{\mathbf{P}\left(\eta_{t} \in \mathcal{M}\right)} \lim _{\Delta \rightarrow 0} \frac{1}{\Delta} \int_{\mathcal{M}} \mathbf{P}\left(\eta_{t+\Delta} \in \mathscr{P} \mid \eta_{t}=\eta\right) \mathbf{P}\left(\eta_{t} \in d \eta\right) \\
=\frac{1}{\mathbf{P}\left(\eta_{t} \in \mathcal{M}\right)} \lim _{\Delta \rightarrow 0} \int_{\mathcal{M}} \frac{1}{\Delta}\left(\mathbf{E}\left(f\left(\eta_{\Delta}\right) / \eta_{0}=\eta\right)-f(\eta)\right) \mathbf{P}\left(\eta_{t} \in d \eta\right) \\
=\frac{1}{\mathbf{P}\left(\eta_{t} \in \mathcal{M}\right)} \mathbf{E} A\left(\eta_{t} ; \mathscr{P}\right) .
\end{gathered}
$$

As the process is ergodic, this yields the desired result.

Example 3.11: Semi-Markov process on a discrete state space. We return to Examples 2.4 and 2.10 and preserve corresponding notations. Let the semi-Markov process by ergodic and let $\pi$ be its stationary distribution of states. Example 2.10 and Proposition 3.10 yield

$$
\lambda_{V}(\infty)=\frac{1}{\pi^{\prime}\left(\mathcal{M}^{\prime}\right)} \sum_{i \in \mathcal{N}} \int_{0}^{\infty} \sum_{j \in \mathscr{T}} A(i, j, y) \pi^{\prime}(i, d y)
$$

where $\pi^{\prime}$ is the stationary distribution of Markov process $\left(\eta_{s}, y_{s}\right)$. It is not difficult to prove (see, for example, Disney and Kiessler [8] (Section 2.7)) that

$$
\pi^{\prime}(i, d y)=K \nu(i) \exp \left(-\int_{0}^{y} h(i, s) d s\right) d y
$$

where function $h$ is defined in Example 2.4, $\nu(\cdot)$ is the stationary distribution of the embedded Markov chain $X$ with transition probabilities $\mathbf{p}(i, j)=\int q(i, j, y) d y$, and $K$ is a norming constant. Let us denote by $\pi$ the stationary distribution of the semi-Markov process. It follows from (3.19) that

$$
\pi(i)=\int_{0}^{\infty} \pi^{\prime}(i, d y)=K \nu(i) \int_{0}^{\infty} \exp \left(-\int_{0}^{y} h(i, s) d s\right) d y=K \nu(i) m(i),
$$


where the mean sojourn time in the state $i, m(i)=\mathbf{E}\left(T_{1} \mid \eta_{0}=i\right)$, is finite. Thus, $\pi$ can be viewed as the stationary distribution of states of a Markov process with the same embedded Markov chain $X$ and the same mean sojourn times $m(i)$. This Markov process has the generator

$$
\mathbf{a}(i, j)=\frac{\mathbf{p}(i, j)}{m(i)}
$$

Substituting (3.19) into (3.18) and applying (2.9) to (2.10), we get

$$
\begin{gathered}
\lambda_{V}(\infty)=\frac{1}{\pi(\mathcal{H})} \sum_{i \in \mathcal{M}} \sum_{j \in \mathscr{P}} \int_{0}^{\infty} p(i, j, y) h(i, y) K \nu(i) \exp \left(-\int_{0}^{y} h(i, s) d s\right) d y \\
=\frac{1}{\pi(\mathcal{H})} \sum_{i \in \mathcal{M}} \sum_{j \in \mathscr{\Phi}} K \nu(i) \int_{0}^{\infty} q(i, j, y) d y \\
=\frac{1}{\pi(\mathcal{H})} \sum_{i \in \mathcal{M}} \sum_{j \in \mathscr{P}} K \nu(i) \mathbf{p}(i, j) \\
=\frac{1}{\sum_{k \in \mathcal{M}} \nu(k) m(k)} \sum_{i \in \mathcal{M}} \sum_{j \in \mathscr{T}} \nu(i) \mathbf{p}(i, j) .
\end{gathered}
$$

This yields

$$
\lambda_{V}(\infty)=\sum_{i \in \mathcal{M}} \frac{\pi(i)}{\pi(\mathcal{M})} \sum_{j \in \mathscr{\Phi}} \frac{\mathbf{p}(i, j)}{m(i)}=\sum_{i \in \mathcal{M}} \frac{\pi(i)}{\pi(\mathcal{M})} \sum_{j \in \mathscr{\Phi}} \mathbf{a}(i, j)
$$

The arguments above imply the following result.

Proposition 3.12: For an ergodic semi-Markov process on a discrete state space, the asymptotic Vesely failure rate is equal to the asymptotic Vesely failure rate of a Markov process having the same embedded Markov chain and the same mean sojourn times in all states.

Remark 3.13: It can be seen from (3.20) that, in the Markovian case, $\lambda_{V}(\infty)=1 / M U T$, where MUT means Mean Up Time of the system (see Pagès and Gondran [20]). The last proposition shows that this is also true in the semi-Markovian case.

Example 3.14: Redundant system. Consider the redundant system described in Examples 2.6 and 2.11 and preserve the same notations. Let us embed $\left(\eta_{s}\right)$ into the Markov process $\left(\eta_{s}, y_{s}\right)$. Let $\pi^{\prime}$ be its stationary distribution of states. By Proposition 3.10 and equation (2.32)

$$
\lambda_{V}(\infty)=\frac{1}{\pi^{\prime}\left(\mathcal{H}^{\prime}\right)} \sum_{\eta \in \mathcal{N}} \int_{0}^{\infty} \sum_{j \in R(\eta)} a^{(i)}(\eta, y) \pi^{\prime}(\eta, d y) .
$$

Since all components of the system are independent, the measure $\pi^{\prime}$ has the product form

$$
\pi^{\prime}(\eta, y)=\prod_{i=1}^{N} \pi^{\prime(i)}(\eta(i), y(i))
$$

and the same is true for the stationary distribution $\pi$ of states of process $\left(\eta_{s}\right)$

$$
\pi(\eta)=\prod_{i=1}^{N} \pi^{(i)}(\eta(i))
$$

For any $i$, function $a^{(i)}$ depends on $\eta(i)$ and $y(i)$ only. Hence, we can write $a^{(i)}(\eta(i), y(i))$ instead of $a^{(i)}(\eta, y)$. Let $\gamma_{i}$ and $\delta_{i}$ be the mean sojourn time of the $i$ th component in "good" state 1 and in "bad" state 0 , respectively, and 


$$
m^{(i)}(\eta(i))= \begin{cases}\gamma_{i}, & \text { if } \eta(i)=1 \\ \delta_{i}, & \text { if } \eta(i)=0\end{cases}
$$

It can be easily seen from (3.19) that

and, consequently,

$$
\pi^{(i)}(\eta(i), y(i))=\frac{1}{\gamma_{i}+\delta_{i}} \exp \left(-\int_{0}^{y(i)} a^{(i)}(\eta(i), s) d s\right)
$$

$$
\int_{0}^{\infty} a^{(i)}(\eta, y) \pi^{\prime}(\eta, d y)=\frac{1}{\gamma_{i}+\delta_{i}} \prod_{j: j \neq i} \frac{m^{(j)}(\eta(j))}{\gamma_{j}+\delta_{j}}=\frac{1}{\gamma_{i}+\delta_{i}} \prod_{j: j \neq i} \pi^{(j)}(\eta(j)) .
$$

Assume additionally that the system is coherent. This means that if the system is operating (resp., failed) and if one more component becomes operating (resp., failed) then the system remains operating (resp., failed). This assumption is typical and natural for reliability studies. Then

$$
\begin{gathered}
\sum_{\eta \in \mathcal{M}} \sum_{i \in R(\eta)} \int_{0}^{\infty} a^{(i)}(\eta, y) \pi^{\prime}(\eta, d y) \\
=\sum_{i} \frac{1}{\gamma_{i}+\delta_{i}} \sum_{\eta: i \in R(\eta)} \prod_{j: j \neq i} \pi^{(j)}(\eta(j)) \\
=\sum_{i} \frac{1}{\gamma_{i}+\delta_{i}}(\pi(\eta:(\eta(1), \ldots, \eta(i-1), 0, \eta(i+1), \ldots, \eta(N)) \in \mathfrak{P}) \\
-\pi(\eta:(\eta(1), \ldots, \eta(i-1), 1, \eta(i+1), \ldots, \eta(N)) \in \mathscr{P})) .
\end{gathered}
$$

Equations (3.21) and (3.22) yield the following formula, well-known to engineers:

$$
\begin{gathered}
\lambda_{V}(\infty)=\frac{1}{\pi(\mathcal{H})} \sum_{i} \frac{1}{\gamma_{i}+\delta_{i}}(\pi(\eta:(\eta(1), \ldots, \eta(i-1), 0, \eta(i+1), \ldots, \eta(N)) \in \mathscr{P}) \\
-\pi(\eta:(\eta(1), \ldots, \eta(i-1), 1, \eta(i+1), \ldots, \eta(N)) \in \mathscr{P})) .
\end{gathered}
$$

It can easily be computed with the help of fault trees and existing software.

The following result generalizes Remark 3.9. It uses PH-distributions (see Asmussen [1] (Section II.6) and Kalashnikov [15] (Subsection 9.5.3)) which can be treated as distributions of absorption times for Markov processes with a finite state space.

Proposition 3.15: Consider the redundant system described in Example 2.6. Let the system be coherent with constant failure rates and general strictly positive repair rates, and repair times having finite means. Then we have:

$$
\lambda^{0}=\lambda_{V}(\infty)
$$

Consequently, if the repair times have PH-distributions or if conditions C0.3 and C0.4 are satisfied, then

$$
\lambda(\infty) \leq \lambda_{V}(\infty)
$$

Proof: We use notations from Examples 2.6 and 2.11. Since failure rates are constant, it is not necessary to consider all $y(i)$ 's from $(2.16)$ to obtain a Markov process. Define another vector $\underline{y}$ with $N$ components: 
Process $\left(\eta_{s}, \underline{y}_{s}\right)$ is Markov with generator

$$
\underline{y}(i)=\left\{\begin{array}{cc}
y(i), & \text { if } \eta(i)=0 \\
0, & \text { if } \eta(i)=1
\end{array}\right.
$$

where

$$
\underline{A} f(\eta, \underline{y})=\sum_{i: \eta(i)=0} \frac{\partial f(\eta, \underline{y})}{\partial y(i)}+\sum_{i} a^{(i)}(\eta(i), \underline{y}(i))\left(f\left(\eta^{(i)}, \underline{y}^{(i)}\right)-f(\eta, y)\right),
$$

$$
a^{(i)}(\eta(i), \underline{y}(i))=\left\{\begin{array}{cl}
\mu^{(i)}(\underline{y}(i)), & \text { if } \eta(i)=0, \\
\lambda^{(i)}(\underline{y}(i))=\lambda^{(i)}(\underline{0})=\lambda^{(i)}, & \text { if } \eta(i)=1 .
\end{array}\right.
$$

All assumptions of Theorem 2.7 are fulfilled and thus

$$
\underline{A}^{0} f(\eta, \underline{y})=\sum_{i: \eta(i)=0} \frac{\partial f(\eta, \underline{y})}{\partial \underline{y}(i)}+\sum_{i \notin R(\eta)} a^{(i)}(\eta(i), \underline{y}(i))\left(f\left(\eta^{(i)}, \underline{y}^{(i)}\right)-f(\eta, \underline{y})\right) .
$$

Let us prove that stationary distrbution $\underline{\pi}^{0}$ of the Markov process with generator $\underline{A}^{0}$ is defined by the relation

$$
\underline{\pi}^{0}(\varphi)=K_{0} \sum_{\eta \in \mathcal{M}} \int \varphi(\eta, \underline{y}) \prod_{j: \eta(j)=0} \exp \left(-\int_{0}^{\underline{y}(j)} \mu^{(j)}(s) d s\right) \prod_{j: \eta(j)=1} \frac{1}{\lambda^{(j)}} \prod_{j: \eta(j)=0} d \underline{y}(j),
$$

where $\varphi$ is a bounded measurable function and $K_{0}>0$ is a norming constant. Define functions $g_{j}$ and $g$ as

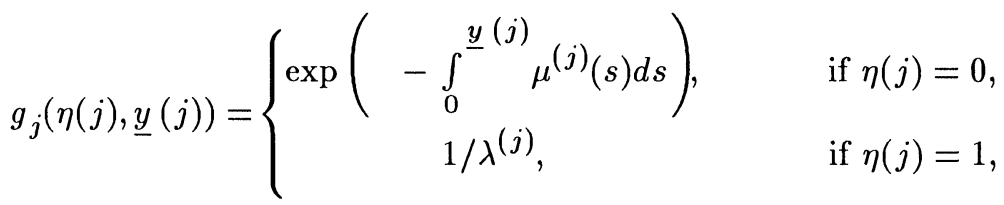

$$
\begin{aligned}
& g(\eta, \underline{y})=\prod_{j} g_{j}(\eta(j), \underline{y}(j)),
\end{aligned}
$$

and prove that, for every function $f$ from the domain of $\underline{A}^{0}$,

$$
C(f) \equiv \sum_{\eta \in \mathcal{M}} \int \underline{A}^{0} f(\eta, \underline{y}) g(\eta, \underline{y}) \prod_{j: \eta(j)=0} d \underline{y}(j)=0 .
$$

Let $i$ be such that $\eta(i)=1$. Then by integrating by parts,

$$
\begin{gathered}
\int \frac{\partial f(\eta, \underline{y})}{\partial \underline{y}(i)} g(\eta, \underline{y}) \prod_{j: \eta(j)=0} d \underline{y}(j) \\
=\int \prod_{j: j \neq i} g_{j}(\eta(j), \underline{y}(j)) \prod_{\substack{j: j \neq i, \eta(j)=0}} d y(j) \int_{0}^{\infty} \frac{\partial f(\eta, \underline{y})}{\partial \underline{y}(i)} \exp \left(-\int_{0}^{\underline{y}(i)} \mu^{(i)}(s) d s\right) d \underline{y}(i) \\
=-\int f\left(\eta, \underline{y}^{(i)}\right) \prod_{j: j \neq i} g_{j}(\eta(j), \underline{y}(j)) \prod_{\substack{j: j \neq i, \eta(j)=0}} d \underline{y}(j)+\int \mu^{(i)}(\underline{y}(i)) f(\eta, \underline{y}) g(\eta, \underline{y}) \prod_{\substack{j: j \neq i, \eta(j)=0}} d \underline{y}(j) .
\end{gathered}
$$

Using the fact that $\underline{y}=\underline{y}^{(i)}$ if $\eta(i)=1$ and system's coherence, we have

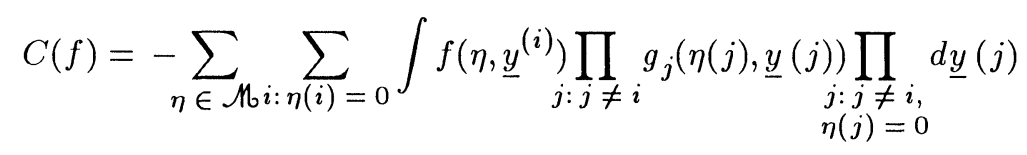




$$
\begin{aligned}
& +\sum_{\eta \in \mathcal{M}} \sum_{i: \eta(i)=0} \int \mu^{(i)}(\underline{y}(i)) f(\eta, \underline{y}) g(\eta, \underline{y}) \prod_{j: \eta(j)=0} d \underline{y}(j) \\
& +\sum_{\eta \in \mathcal{M}} \sum_{i: \eta(i)=0} \int f\left(\eta^{(i)}, \underline{y}^{(i)}\right) \prod_{j: j \neq i} g_{j}(\eta(j), \underline{y}(j)) \prod_{\substack{j: j \neq i, \eta(j)=0}} d \underline{y}(j) \\
& -\sum_{\eta \in \mathcal{M}} \sum_{i: \eta(i)=0} \int \mu^{(i)}(\underline{y}(i)) f(\eta, \underline{y}) f(\eta, \underline{y}) g(\eta, \underline{y}) \prod_{j: \eta(j)=0} d \underline{y}(j) \\
& +\sum_{\eta \in \mathcal{M}} \sum_{\substack{i: \eta^{(i)} \in \mathcal{M}, \eta(i)=1}} \int f\left(\eta^{(i)}, \underline{y}^{(i)}\right) \prod_{j: j \neq i} g_{j}(\eta(j), \underline{y}(j)) \prod_{\substack{j: j \neq i, \eta(j)=0}} d \underline{y}(j) \\
& -\sum_{\eta \in \mathcal{M}} \sum_{\substack{i: \eta^{(i)} \in \mathcal{N}, \eta(i)=1}} \int f\left(\eta, \underline{y}^{(i)}\right) \prod_{j: j \neq i} g_{j}(\eta(j), \underline{y}(j)) \prod_{\substack{j: j \neq i, \eta(j)=0}} d y(j) \\
& =-\sum_{\eta \in \mathcal{H}} \sum_{i: \eta} \int f\left(\eta, \underline{y}^{(i)}\right) \prod_{j: j \neq i} g_{j}(\eta(j), \underline{y}(j)) \prod_{\substack{j: j \neq i, \eta(j)=0}} d \underline{y}(j) \\
& +\sum_{\eta \in \mathcal{N}_{i: \eta}} \sum_{(i) \in \mathcal{M}} \int f\left(\eta^{(i)}, \underline{y}^{(i)}\right) \prod_{j: j \neq i} g_{j}(\eta(j), \underline{y}(j)) \prod_{\substack{j: j \neq i, \eta(j)=0}} d \underline{y}(j) \\
& =0 \text {. }
\end{aligned}
$$

Similar calculations show that the stationary probability distribution for process $\left(\eta_{s}, \underline{y}_{s}\right)$ is given by the relation

$$
\underline{\pi}(\varphi)=K \sum_{\eta \in E} \int \varphi(\eta, \underline{y}) g(\eta, \underline{y}) \prod_{j: \eta(j)=0} d \underline{y}(j),
$$

where $\varphi$ is a bounded measurable function and $K>0$ is a norming constant.

Equations (3.23), (3.24), (3.25), and (3.26) imply that

$$
\underline{\pi}^{0}(\cdot)=\frac{\underline{\pi}(\cdot)}{\underline{\pi}\left(\mathcal{M} \times \mathbf{R}_{+}^{N}\right)} .
$$

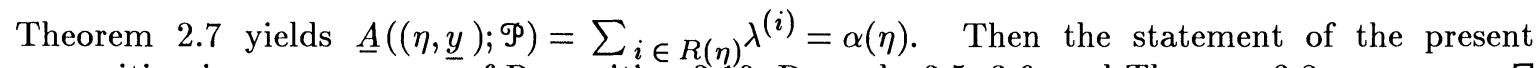
proposition is a consequence of Proposition 3.10, Remarks 3.5, 3.6, and Theorem 3.2.

Remark 3.16: Conditions of Proposition 3.15 are optimal in the following sense. Generally, $\lambda_{V}(\infty) \neq \lambda^{0}$ for a redundant system where failure rates are not constant. Moreover, the inequality $\lambda_{V}(\infty)<\lambda(\infty)$ may hold true. As an example, let us consider two independent components $\mathrm{C} 1$ and $\mathrm{C} 2$ in parallel. Suppose that the lifetime of component C1 has the Erlang distribution with parameters 2 and 1 (that is the lifetime can be viewed as a sum of two independent random variables, each one being exponentially distributed with parameter 1) and its repair rate is $\mu^{(1)}=10$. Suppose that the failure rate of component C2 is $\lambda^{(2)}=1$ and its repair rate is $\mu^{(2)}=10$. It can be seen that

$$
\lambda(\infty)=8.7024 \cdot 10^{-2}, \quad \lambda_{V}(\infty)=8.6957 \cdot 10^{-2}, \quad \lambda^{0}=9.4340 \cdot 10^{-2},
$$

that is, $\lambda^{0} \neq \lambda_{V}(\infty)$ and $\lambda_{V}(\infty)<\lambda(\infty)$. 


\section{A Stationary Representation of the Asymptotic Failure Rate}

Throughout the remainder of this paper we will assume that the following Conditions $C 1$ are met:

\section{Conditions C1}

1. Conditions $\mathrm{C} 0$ hold.

2. Intensity $\alpha_{u}$ depends on the current state $\eta_{u}$ only, that is $\alpha_{u}=\alpha\left(\eta_{u}^{0}\right)$.

In this section, we construct a regenerative process $\left(\tilde{\eta}_{s}\right)$ such that $\lambda(\infty)$ is equal to the stationary mean value of $\alpha\left(\tilde{\eta}_{s}\right)$. For this, we define the following measure on subsets of $\mathcal{M}$ :

$$
\tilde{\pi}(\cdot)=\frac{\mathbf{E} \int_{0}^{S} I\left(\eta_{t}^{0} \in \cdot\right) \exp \left(-\int_{0}^{t}\left(\alpha_{u}-\kappa\right) d u\right) d t}{\mathbf{E} \int_{0}^{S} \exp \left(-\int_{0}^{t}\left(\alpha_{u}-\kappa\right) d u\right) d t} .
$$

Then, by (3.3), $\lambda(\infty)$ can be represented as

$$
\lambda(\infty)=\int_{\mathcal{M}} \alpha(\eta) \tilde{\pi}(d \eta)
$$

Remark 4.1: It can be easily seen (using (2.2) and the same arguments as in the proof of Theorem 3.2) that $\tilde{\pi}$ is the quasi-stationary distribution of process $\left(\eta_{s}\right)$, i.e.,

$$
\tilde{\pi}(\cdot)=\lim _{t \rightarrow \infty} \mathbf{P}\left(\eta_{t} \in \cdot \mid \sigma>t\right) .
$$

Before constructing a regenerative process with stationary distribution $\tilde{\pi}$, let us build a regenerative process $\eta^{1} \equiv\left(\eta_{s}^{1}\right)$ with initial distribution $\tilde{\pi}$.

Formula (4.1) yields the following general construction. Let us regard random process $\eta^{0} \equiv\left(\eta_{s}^{0}\right)(\omega)$ as defined on probability space $(\Omega, \mathcal{F}, \mathbf{P})$ and expand this space as follows (a similar construction was used by Thorisson [22]; see also Kalashnikov [16] (Section 1.6)). Let $\mathscr{B}$ be the collection of all Borel subsets of $[0,1]$. Set

where

$$
\begin{gathered}
\omega^{\prime}=(\omega, u), \omega \in \Omega, u \in[0,1] \\
\Omega^{\prime}=\Omega \times[0,1] ; \quad F^{\prime}=\mathscr{F} \times \mathfrak{B} ; \\
\mathbf{P}^{\prime}\left(d \omega^{\prime}\right)=\mathbf{P}^{\prime}(d \omega, d u)=\mathbf{P}(d \omega) d u ; \\
\mathbf{P}^{\prime \prime}\left(d \omega^{\prime}\right)=\frac{1}{D} S(\omega) \exp \left(-\int_{0}^{u S(\omega)}\left(\alpha\left(\eta_{v}^{0}(\omega)\right)-\kappa\right) d v\right) \mathbf{P}^{\prime}\left(d \omega^{\prime}\right),
\end{gathered}
$$

$$
D=\mathbf{E} \int_{0}^{S} \exp \left(-\int_{0}^{t}\left(\alpha\left(\eta_{v}^{0}\right)-\kappa\right) d v\right) d t .
$$

This construction has the following meaning. Formulas (4.3) and (4.4) imply that the r.v. $U$ is defined independently of other random elements (this leads to the probability $\mathbf{P}^{\prime}$ ). Then we introduce a new probability measure $\mathbf{P}^{\prime \prime}$ on the expanded probability space (see (4.5) and (4.6)), where $D$ is a norming constant. After this, we can define a new process $\eta^{1} \equiv\left(\eta_{s}^{1}\right)$ on the probability space $\left(\Omega^{\prime}, \mathcal{F}^{\prime}, \mathbf{P}^{\prime \prime}\right)$ by the relation

$$
\eta_{t}^{1}\left(\omega^{\prime}\right)=\eta_{t+u S(\omega)}^{0}(\omega) \equiv\left(\theta_{u S(\omega)} \eta^{0}\right)_{t}, \quad \omega^{\prime}=(\omega, u)
$$

where $\theta_{s}$ stands for the shift operator on time $s$ onward. We are going to see that construction 
(4.3) through (4.7) implies that process $\eta^{1}$ can be viewed as regenerative with regeneration times

Consider the pair

$$
S_{k}^{1}\left(\omega^{\prime}\right)=S_{k+1}^{0}(\omega)-S(\omega) u, \quad k \geq 0 .
$$

$$
\left(\eta^{1}, S^{1}\right)\left(\omega^{\prime}\right)=\left(\left(\eta_{s}^{1}\right), S_{0}^{1}, S_{1}^{1}, \ldots\right)\left(\omega^{\prime}\right)
$$

defined by (4.7) and (4.8). Then the following chain of relations holds

$$
\begin{gathered}
\mathbf{P}^{\prime \prime}\left(\theta_{S_{k}^{1}}\left(\eta^{1}, S^{1}\right)\left(\omega^{\prime}\right) \in \cdot\right) \\
=\frac{1}{D} \int_{\Omega} S(\omega) \int_{0}^{1} I\left(\theta_{S_{k+1}^{0}(\omega)-u S(\omega)} \theta_{u S(\omega)}\left(\eta^{0}, S^{0}\right)(\omega) \in \cdot\right) \\
\times \exp \left(-\int_{0}^{u S(\omega)}\left(\alpha\left(\eta_{v}^{0}\right)-\kappa\right) d v\right) d u \mathbf{P}(d \omega) \\
=\frac{1}{D} \int_{\Omega} I\left(\theta_{S_{k+1}^{0}(\omega)}\left(\eta^{0}, S^{0}\right)(\omega) \in \cdot\right) \int_{0}^{S(\omega)} \exp \left(-\int_{0}^{t}\left(\alpha\left(\eta_{v}^{0}\right)-\kappa\right) d v\right) d t \mathbf{P}(d \omega) \\
=\mathbf{E} I\left(\theta_{S_{k+1}^{0}(\omega)}\left(\eta^{0}, S^{0}\right)(\omega) \in \cdot\right) \frac{1}{D} \mathbf{E} \int_{0}^{t} \exp \left(-\int_{0}^{t}\left(\alpha\left(\eta_{v}^{0}\right)-\kappa\right) d v\right) d t \\
=\mathbf{E} I\left(\theta_{S_{k+1}^{0}(\omega)}\left(\eta^{0}, S^{0}\right)(\omega) \in \cdot\right) \\
=\mathbf{E} I\left(\left(\eta^{0}, S^{0}\right)(\omega) \in \cdot\right) .
\end{gathered}
$$
Therefore, all shifts $\theta_{S_{k}^{1}}\left(\eta^{1}, S^{1}\right)$ are identically distributed. Quite similarly, one can prove that
events

and

$$
\left\{\theta_{S_{k}^{1}}\left(\eta^{1}, S^{1}\right) \in \cdot\right\}
$$

$$
\left\{\left(S_{0}^{1}, \ldots, S_{k}^{1}\right) \in \cdot\right\}
$$

are independent. Moreover, it follows from (4.1), (4.5), and(4.7) that the initial distribution of process $\eta^{1}$ is $\tilde{\pi}$.

Remark 4.2: Equality (4.9) shows that all shifts $\theta_{S_{k}^{1}}\left(\eta^{1}, S^{1}\right)$ are distributed as $\left(\eta^{0}, S^{0}\right)$. By the terminology adopted in the theory of regenerative processes, this means that $\left(\eta^{1}, S^{1}\right)$ is a version of $\left(\eta^{0}, S^{0}\right)$, i.e., these two processes have identically distributed cycles but perhaps different delays. Regenerative process $\left(\eta^{1}, S^{1}\right)$ is delayed, in general; that is, $\mathbf{P}\left(S_{0}^{1} \neq 0\right)>0$.

Remark 4.3: If $\eta^{0}$ is a Markov process, then the above construction shows that $\eta^{1}$ is also a Markov process with the same transition probabilities as $\eta^{0}$ (and with the initial distribution $\tilde{\pi}$ ).

We now construct a regenerative process $\tilde{\eta}$ that has $\tilde{\pi}$ as its stationary distribution. For this, let us introduce processes $\eta^{i}, i \geq 1$ and positive random variables $T^{i}$ such that

- $\quad$ processes $\eta^{i}, i \geq 0$ are independent;

- $\quad$ process $\eta^{0}$ is a probabilistic replica of the "original" process $\eta^{0}$ in the sense that it has the same probability law (because of this, we did not change its notation);

- $\quad$ processes $\eta^{i}, i \geq 1$ are i.i.d. probabilistic replicas of the "original" process $\eta^{1}$;

- $\quad$ for any $i \geq 0$, 


$$
\mathbf{P}\left(T^{i} \leq t \mid\left(\eta_{s}^{i}\right)_{s \geq 0}\right)=\mathbf{P}\left(T^{i} \leq t \mid\left(\eta_{s}^{i}\right)_{0} \leq s \leq t\right)=1-\exp \left(-\int_{0}^{t} \alpha\left(\eta_{u}^{i}\right) d u\right)
$$

Let us denote by $\left(S_{k}^{i}\right)_{k} \geq 0$ regeneration times of process $\eta^{i}$. Recall that $S_{0}^{i} \neq 0$ in general (see Remark 4.2).

Hereafter, let us regard all processes as defined on the same measurable space $\left(\Omega^{\prime}, \mathcal{F}^{\prime}\right)$ and preserve notations $\mathbf{P}, \mathbf{P}^{\prime}$, and $\mathbf{P}^{\prime \prime}$ for the measures indicated in (4.4) and (4.5). Construct process $\widetilde{\eta}$ from fragments of the regenerative process $\eta^{0}$ and its i.i.d. versions $\eta^{i}, i \geq 1$, as follows:

$$
\tilde{\eta}_{t}\left(\omega^{\prime}\right)=\left\{\begin{array}{cc}
\eta_{t}^{0}(\omega), & \text { if } t<T^{0} \\
\eta_{t-\left(T^{0} T+\ldots+T^{i-1}\right)}^{i}\left(\omega^{\prime}\right), & \text { if } T^{0}+\ldots+T^{i-1} \leq t<T^{0}+\ldots+T^{i}, i \geq 1
\end{array}\right.
$$

Then for each $i \geq 1$, set $\widetilde{S}^{i}=\left\{T^{-}+\ldots+T^{i-1}+S_{k}^{i}: k \geq 0,0 \leq S_{k}^{i}<T^{i}\right\}$ is the collection of regeneration times of the process $\eta_{t-T^{0}-\ldots-T^{i-1}}^{i}$ occurring before its stopping at time $T^{i}$. Set $\tilde{S}^{i}$ may be empty if $T^{i} \leq S_{0}^{i}$. Process $\tilde{\eta}$ thus defined can be viewed as regenerative in at least two different ways:

(i) with regeneration times $\left(T^{0}+\ldots+T^{k}\right)_{k \geq 0}$;

(ii) with regeneration times $\widetilde{S}=\left(\widetilde{S}^{0}, \widetilde{S}^{1}{\underset{\widetilde{S}}{ }}^{2}, \ldots\right)=\left(\widetilde{S}_{0}, \widetilde{S}_{1}, \ldots\right)$ consisting of all "occurred" regeneration times. By construction, $\widetilde{S}_{0}=0$.

Let us call the regeneration times indicated above as those of the first and second types, respectively.

Theorem 4.4: Under assumptions $C 1$, there exists a regenerative process $\tilde{\eta}$ with stationary distribution $\tilde{\pi}$ such that

Moreover,

$$
\lambda(\infty) \int_{\mathcal{M}} \alpha(\eta) \tilde{\pi}(d \eta)
$$

$$
\tilde{\eta}_{t}=\eta_{t}^{0} \text { if } t<T^{0}, \mathbf{P}\left(\tilde{\eta}_{T^{0}} \in \cdot\right)=\tilde{\pi}(\cdot),
$$

where $T^{0}$ is an r.v. such that

$$
\mathbf{P}\left(T^{0} \leq t \mid\left(\eta_{s}^{0}\right)_{s} \geq 0\right)=\mathbf{P}\left(T^{0} \leq t \mid\left(\eta_{s}^{0}\right)_{0 \leq s \leq t}\right)=1-\exp \left(-\int_{0}^{t} \alpha\left(\eta_{u}^{0}\right) d u\right) .
$$

Proof: Let us consider the regenerative process $(\widetilde{\eta}, \widetilde{S})$. In order not to introduce new notation, we denote by $\tilde{\pi}$ the stationary distribution of $\widetilde{\eta}$ which can be written as

$$
\tilde{\pi}(\cdot)=\frac{1}{\mathbf{E} \widetilde{S}_{1}} \mathbf{E} \int_{0}^{\widetilde{S}_{1}} I\left(\tilde{\eta}_{t} \in \cdot\right) d t
$$

To prove that distributions (4.1) and (4.12) are the same, it is sufficient to state that

$$
N \equiv \mathbf{E} \int_{0}^{\tilde{S}_{1}} I\left(\tilde{\eta}_{t} \in \cdot\right) d t=\mathbf{E} \int_{0}^{S} I\left(\eta_{t}^{0} \in \cdot\right) \exp \left(-\int_{0}^{t}\left(\alpha\left(\eta_{u}^{0}\right)-\kappa\right) d u\right) d t
$$

By construction (4.11), 


$$
\begin{aligned}
N & =\mathbf{E} \int_{0}^{\widetilde{S}_{1} \wedge T^{0}} I\left(\tilde{\eta}_{t} \in \cdot\right) d t+\mathbf{E}\left(I\left(T^{0}<\widetilde{S}_{1}\right) \int_{T^{0}}^{\widetilde{S}_{1}} I\left(\widetilde{\eta}_{t} \in \cdot\right) d t\right) \\
& =\mathbf{E} \int_{0}^{S_{1}^{0}} I\left(\eta_{t}^{0} \in \cdot\right) \exp \left(-\int_{0}^{t} \alpha\left(\eta_{u}^{0}\right) d u\right) d t+\mathbf{P}\left(T^{0}<\widetilde{S}^{1}\right) N_{1}
\end{aligned}
$$

where

$$
N_{1}=\mathbf{E} \int_{0}^{S_{1}^{\prime}} I\left(\theta_{T^{0}} \tilde{\eta}_{t} \in \cdot\right) d t
$$

and $S_{1}^{\prime}$ is the first regeneration time of the second type for the shifted process $\theta_{T_{0}} \tilde{\eta}$; evidently, $S_{1}^{\prime}>T_{0}$. Since $S_{1}^{0}$ is measurable with respect to $\left(\eta_{s}^{0}\right)$, it can be regarded as a constant when conditioning with respect to $\left(\eta_{s}^{0}\right)$. Therefore, equality $(4.10)$ yields

$$
\mathbf{P}\left(T^{0}<\widetilde{S}_{1}\right)=\mathbf{P}\left(T^{0}<S_{1}^{0}\right)=\left(1-\operatorname{Eexp}\left(-\int_{0}^{S_{1}^{0}} \alpha\left(\eta_{u}^{0}\right) d u\right)\right)
$$

All shifts $\theta_{T^{0}+\ldots+T^{k}} \tilde{\eta}$ are identically distributed. Because of this we have, similarly to (4.14),

Therefore,

$$
N_{1}=\mathbf{E}^{\prime \prime} \int_{0}^{S_{0}^{1}} I\left(\eta_{t}^{1} \in \cdot\right) \exp \left(-\int_{0}^{t} \alpha\left(\eta_{v}^{1}\right) d v\right) d t+\left(1-\mathbf{E}^{\prime \prime} \exp \left(-\int_{0}^{S_{0}^{1}} \alpha\left(\eta_{t}^{1}\right) d t\right)\right) N_{1} \text {. }
$$

$$
N_{1}=\frac{\mathbf{E}^{\prime \prime} \int_{0}^{S_{0}^{1}} I\left(\eta_{t}^{1} \in \cdot\right) \exp \left(-\int_{0}^{t} \alpha\left(\eta_{v}^{1}\right) d v\right) d t}{\mathbf{E}^{\prime \prime} \exp \left(-\int_{0}^{S_{0}^{1}} \alpha\left(\eta_{t}^{1}\right) d t\right)} .
$$

Let us use construction (4.7) of process $\eta^{1}$ in order to find $N_{1}$. By (4.3) to (4.8) and changing variables, we have

$$
\begin{gathered}
\mathbf{E}^{\prime \prime} \int_{0}^{S_{0}^{1}} I\left(\eta_{t}^{1} \in \cdot\right) \exp \left(-\int_{0}^{t} \alpha\left(\eta_{v}^{1}\right) d v\right) d t \\
=\frac{1}{D} \int_{\Omega} S(\omega) \int_{0}^{1} \exp \left(-\int_{0}^{u S(\omega)}\left(\alpha\left(\eta_{v}^{0}(\omega)\right)-\kappa\right) d v\right) \\
\times \int_{0}^{S(\omega)(1-u)} I\left(\eta_{t+u S(\omega)}^{0} \in \cdot\right) \exp \left(-\int_{0}^{t} \alpha\left(\eta_{v+u S(\omega)}^{0}(\omega)\right) d v\right) d t d u \mathbf{P}(d \omega) \\
=\frac{1}{D} \mathbf{E} \int_{0}^{S} \exp \left(-\int_{0}^{s}\left(\alpha\left(\eta_{v}^{0}\right)-\kappa\right) d v\right) \int_{0}^{S-s} I\left(\eta_{t+s}^{0} \in \cdot\right) \exp \left(-\int_{0}^{t} \alpha\left(\eta_{v+s}^{0}\right) d v\right) d t d s
\end{gathered}
$$


522

CHRISTIANE COCOZZA-THIVENT and VLADIMIR KALASHNIKOV

$$
\begin{aligned}
& =\frac{1}{D} \mathbf{E} \int_{0}^{S} I\left(\eta_{u}^{0} \in \cdot\right) \exp \left(-\int_{0}^{u} \alpha\left(\eta_{v}^{0}\right) d v\right) \int_{0}^{u} e^{\kappa s} d s d u \\
& =\frac{1}{\kappa D} \mathbf{E} \int_{0}^{S} I\left(\eta_{u}^{0} \in \cdot\right) \exp \left(-\int_{0}^{u} \alpha\left(\eta_{v}^{0}\right) d v\right)\left(e^{\kappa u}-1\right) d u .
\end{aligned}
$$

Similarly, by (3.2),

$$
\begin{gathered}
\mathbf{E}^{\prime \prime} \exp \left(-\int_{0}^{S_{0}^{1}} \alpha\left(\eta_{t}^{1}\right) d t\right)=\frac{1}{\kappa D} \mathbf{E} \exp \left(-\int_{0}^{S} \alpha\left(\eta_{v}^{0}\right) d v\right)\left(e^{\kappa S}-1\right) \\
=\frac{1}{\kappa D}\left(1-\mathbf{E} \exp \left(-\int_{0}^{S} \alpha\left(\eta_{v}^{0}\right) d v\right)\right) .
\end{gathered}
$$

Substituting this into (4.16), we have

$$
N_{1}=\frac{\mathbf{E} \int_{0}^{S} I\left(\eta_{u}^{0} \in \cdot\right) \exp \left(-\int_{0}^{u} \alpha\left(\eta_{v}^{0}\right) d v\right)\left(e^{\kappa u}-1\right) d u}{1-\mathbf{E} \exp \left(-\int_{0}^{S} \alpha\left(\eta_{v}^{0}\right) d v\right)} .
$$

It follows from (4.14), (4.15), and (4.19) that

$$
N=\mathbf{E} \int_{0}^{S} I\left(\eta_{t}^{0} \in \cdot\right) \exp \left(-\int_{0}^{t}\left(\alpha\left(\eta_{u}^{0}\right)-\kappa\right) d u\right) d t .
$$

Hence, relation (4.13) holds true and Theorem 4.4 is proved.

Remark 4.5: The construction above is a generalization of the construction from CocozzaThivent and Roussignol [4] proposed there for Markov processes on a discrete state space. Let us notice that Conditions C1 are met for such processes (see Remark 3.5) except for Assumption C0.4 which is not needed.

It follows from Theorem 4.4 that

$$
\lambda(\infty)=\frac{1}{\mathbf{E} \widetilde{S}_{1}} \mathbf{E} \int_{0}^{\widetilde{S}_{1}} \alpha\left(\widetilde{\eta}_{t}\right) d t .
$$

Now, let us give another expression for $\lambda(\infty)$ in terms of processes $\eta^{0}$ and $\eta^{1}$. Similarly to (4.14) and (4.16),

$$
\begin{gathered}
\mathbf{E} \int_{0}^{\widetilde{S}_{1}} \alpha\left(\widetilde{\eta}_{t}\right) d t=\mathbf{E} \int_{0}^{S} \alpha\left(\eta_{t}^{0}\right) \exp \left(-\int_{0}^{t} \alpha\left(\eta_{u}^{0}\right) d u\right) d t \\
+\left(1-\mathbf{E} \exp \left(-\int_{0}^{S} \alpha\left(\eta_{t}^{0}\right) d t\right)\right) \frac{\mathbf{E}^{\prime \prime} \int_{0}^{S_{0}^{1}} \alpha\left(\eta_{t}^{1}\right) \exp \left(-\int_{0}^{t} \alpha\left(\eta_{u}^{1}\right) d u\right) d t}{\mathbf{E}^{\prime \prime} \exp \left(-\int_{0}^{S_{0}^{1}} \alpha\left(\eta_{t}^{1}\right) d t\right)} \\
=\frac{1-\mathbf{E} \exp \left(-\int_{0}^{S} \alpha\left(\eta_{t}^{0}\right) d t\right)}{\mathbf{E}^{\prime \prime} \exp \left(-\int_{0}^{S_{0}^{1}} \alpha\left(\eta_{t}^{1}\right) d t\right)}
\end{gathered}
$$


and

$$
\begin{gathered}
\mathbf{E}^{\prime \prime} \widetilde{S}_{1}=\mathbf{E} \int_{0}^{S} \exp \left(-\int_{0}^{t} \alpha\left(\eta_{u}^{0}\right) d u\right) d t \\
+\frac{\left(1-\mathbf{E} \exp \left(-\int_{0}^{S} \alpha\left(\eta_{t}^{0}\right) d t\right)\right) \mathbf{E}^{\prime \prime} \int_{0}^{S_{0}^{1}} \exp \left(-\int_{0}^{t} \alpha\left(\eta_{u}^{1}\right) d u\right) d t}{\mathbf{E}^{\prime \prime} \exp \left(-\int_{0}^{S_{0}^{1}} \alpha\left(\eta_{t}^{1}\right) d t\right)} .
\end{gathered}
$$

Therefore,

where

$$
\begin{gathered}
\lambda(\infty)=\frac{1}{D_{1}}\left(1-\mathbf{E} \exp \left(-\int_{0}^{S} \alpha\left(\eta_{t}^{0}\right) d t\right)\right) \\
D_{1}=\mathbf{E} \int_{0}^{S} \exp \left(-\int_{0}^{t} \alpha\left(\eta_{u}^{0}\right) d u\right) d t \mathbf{E}^{\prime \prime} \exp \left(-\int_{0}^{S_{0}^{1}} \alpha\left(\eta_{t}^{1}\right) d t\right) \\
+\left(1-\mathbf{E} \exp \left(-\int_{0}^{S} \alpha\left(\eta_{t}^{0}\right) d t\right)\right) \mathbf{E}^{\prime \prime} \int_{0}^{S_{0}^{1}} \exp \left(-\int_{0}^{t} \alpha\left(\eta_{u}^{1}\right) d u\right) d t
\end{gathered}
$$

The expectations $\mathbf{E}^{\prime \prime} \exp \left(-\int_{0}^{S_{0}^{1}} \alpha\left(\eta_{t}^{1}\right) d t\right)$ and $\mathbf{E}^{\prime \prime} \int_{0}^{S_{0}^{1}} \exp \left(-\int_{0}^{t} \alpha\left(\eta_{u}^{1}\right) d u\right) d t$ can be expressed in terms of "ordinary" expectations E with the help of formulas (4.17) and (4.18) or Remark 4.3.

The estimates derived above can be used conveniently owing to the fact that, in many practical problems, one can estimate all terms involved in (4.21) and (4.22) and thus give bounds for $\lambda(\infty)$ as we will see it in the following sections.

\section{Accuracy Estimates of the Asymptotic Failure Rate}

In this section, we obtain accuracy estimates for the proposed approximations of the asymptotic failure rate.

\subsection{Accuracy of $\lambda^{0}$}

Let $\lambda^{0}$ be defined as in (3.9). Suppose that $\alpha_{u}=\alpha\left(\eta_{u}^{0}\right)$ and the assumptions of Conditions C0 or Remark 3.5 are satisfied. Then

$$
\lambda^{0}=\frac{\mathbf{E} \int_{0}^{S} \alpha\left(\eta_{u}^{0}\right) d u}{\mathbf{E} S}
$$

We have already proved, in Proposition 3.3 , that $\lambda^{0} \geq \lambda(\infty)$. Let us estimate the relative accuracy of this approximation:

By (4.21), (4.22), and (5.1),

$$
\rho^{0}=\frac{\lambda^{0}-\lambda(\infty)}{\lambda(\infty)}
$$

$$
\rho^{0}=\frac{D_{1}}{\mathbf{E} S} \frac{\mathbf{E} \int_{0}^{S} \alpha\left(\eta_{u}^{0}\right) d u}{1-\mathbf{E} \exp \left(-\int_{0}^{S} \alpha\left(\eta_{u}^{0} d u\right)\right.}-1
$$


Denote

Routine estimates show that

$$
\xi=\int_{0}^{S} \alpha\left(\eta_{u}^{0}\right) d u
$$

and

$$
D_{1} \leq \mathbf{E} S+\mathbf{E} \xi \mathbf{E} S_{0}^{1}
$$

$$
1-\mathbf{E} \exp \left(-\int_{0}^{S} \alpha\left(\eta_{u}^{0}\right) d u\right) \geq \mathbf{E} \xi-\frac{1}{2} \mathbf{E} \xi^{2}
$$

Bringing together (5.1) through (5.6) and assuming that $\mathbf{E} \xi^{2}<2 \mathbf{E} \xi$, we arrive at the inequality

$$
\rho^{0} \leq \frac{\mathbf{E} \xi \mathbf{E} S_{0}^{1} / \mathbf{E} S+\mathbf{E} \xi^{2} / 2 \mathbf{E} \xi}{1-\mathbf{E} \xi^{2} / 2 \mathbf{E} \xi}
$$

In practice, all relevant characteristics in (5.7) can be estimated. Namely, ES represents the mean inter-regeneration time for process $\left(\eta_{s}^{0}\right) ; \mathbf{E} S_{0}^{1}$ is the mean length of the delay of process $\left(\eta_{s}^{1}\right)$; and $\xi$ is the integral intensity to fail accumulated during a regeneration cycle of $\left(\eta_{s}^{0}\right)$. All these quantities can be estimated, say, by the test functions method (see Kalashnikov [15] (Chapter 50)) which uses rather general information about processes under investigation and does not require solving various equations. Corresponding examples will be given in [6]. We only note that both summands in the numerator on the right-hand side of (5.7) are small as a rule for highly reliable systems.

\subsection{Accuracy of $\lambda_{V}(\infty)$}

Let us estimate the relative accuracy

$$
\rho_{V}=\frac{\left|\lambda_{V}(\infty)-\lambda(\infty)\right|}{\lambda(\infty)}
$$

For this, let us give a stationary representation for $\lambda_{V}(\infty)$ similar to that presented in Theorem 4.4. We know that (see (3.17))

Let

$$
\lambda_{V}(\infty)=\frac{1}{\pi(\mathcal{H})} \int_{\mathcal{M}} A(\eta ; \Im) \pi(d \eta) .
$$

$$
\pi^{\prime}(\cdot)=\frac{\pi(\cdot)}{\pi(\mathscr{M})}
$$

be the conditional stationary measure of $\left(\eta_{s}\right)$ restricted to $\mathcal{N}$. Let us prove that $\pi^{\prime}$ can be regarded as the stationary measure of a regenerative process $\eta^{\prime} \equiv\left(\eta_{s}^{\prime}\right)$ and show that $\eta^{\prime}$ can be constructed from $\eta^{0}$ and its i.i.d. versions similarly to process $\widetilde{\eta}$ (see (4.10) and (4.11)).

Assume that the underlying process $\left(\eta_{s}\right)$ is stationary. Define the following r.v.'s (which are stopping times for $\left.\left(\eta_{s}\right)\right)$ :

$$
\begin{gathered}
V_{0}=\min \left\{t: \eta_{t} \in \mathscr{\Phi}, t \geq 0\right\}, \\
U_{k}=\min \left\{t: \eta_{t} \in \mathcal{M}, t>V_{k}\right\}, k \geq 0 \\
V_{k}=\min \left\{t: \eta_{t} \in \mathscr{P}, t>U_{k-1}\right\}, k \geq 1 .
\end{gathered}
$$

Let

$$
\eta(k)=\eta_{U_{k}}, \quad k \geq 0
$$

Evidently, $\left(\eta(k), U_{k}\right)_{k \geq 0}$, is a Markov renewal process. 
Theorem 5.1: Let $\left(\eta_{s}\right)$ be an ergodic Markov process satisfying the assumptions of Proposition 3.10 and having stationary distribution $\pi(\cdot)$. Then there exists a regenerative process $\eta^{\prime}$ with stationary distribution $\pi^{\prime}$ satisfying $\pi^{\prime}(\cdot)=\pi(\cdot) / \pi(\mathcal{M})$, and

Moreover,

$$
\lambda_{V}(\infty)=\int_{\mathcal{M}} \alpha(\eta) \pi^{\prime}(d \eta)
$$

where $T^{0}$ is a r.v. such that

$$
\eta_{t}^{\prime}=\eta_{t}^{0} \text { if } t<T^{0}, \mathbf{P}\left(\eta_{T^{0}}^{\prime} \in \cdot\right)=p(\cdot)
$$

$$
\mathbf{P}\left(T^{0} \leq t \mid\left(\eta_{s}^{0}\right)_{s \geq 0}\right)=\mathbf{P}\left(T^{0} \leq t \mid\left(\eta_{s}^{0}\right)_{0} \leq s \leq t\right)=1-\exp \left(-\int_{0}^{t} \alpha\left(\eta_{u}^{0}\right) d u\right) .
$$

Proof: Since $\left(\eta_{s}\right)$ has a stationary distribution, $\eta(k), k>1$, has a stationary distribution as well. Without loss of generality we can regard the stationary version of process $\left(\eta_{s}\right)$ as defined on $-\infty<s<\infty$ and similarly we can define sequences $U_{k}$ and $V_{k}$ for negative values of $k$. By stationarity of $\left(\eta_{s}\right)$, the sequence $\left(\left(\eta_{s}\right)_{U_{k-1}}<s \leq U_{k}, U_{k}\right)$ is a stationary marked process that is

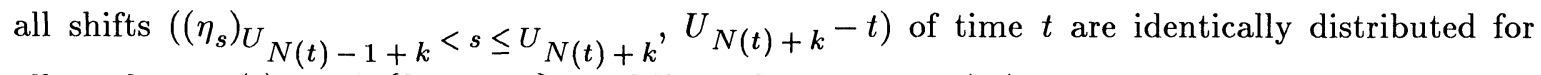
all $t$, where $N(t)=\min \left\{k: U_{k}>t\right\}$. It follows that sequence $\left(\eta_{s}\right)_{U_{k-1}}<s \leq U_{k}, k \geq 1$ is stationary and has the corresponding Palm distribution (see Franken, König, Arndt and Schmidt [12] (Theorem 1.3.1)). Hence, $\eta(k)$ is stationary. Denote its unique stationary distribution by $p(\cdot)$.

Viewing $\left(\eta_{s}\right)$ as a semi-regenerative process with semi-regeneration times $U_{k}, k \geq 0$ (see Disney and Kiessler [8] and Çinlar [2] (Chapter 10, Section 6)), we can represent $\pi$ in the form

$$
\pi(\cdot)=\frac{\mathbf{E} \int_{0}^{\tau} I\left(\eta_{t}^{p} \in \cdot\right) d t}{\mathbf{E} \tau}
$$

In formula $(5.9),\left(\eta_{s}^{p}\right)$ is a version of the Markov process $\left(\eta_{s}\right)$ having the initial distribution $p(\cdot)$ and $\tau$ is the length of a semi-regeneration cycle of this version, that is

where

$$
\tau=\sigma+\sigma_{r}
$$

$$
\sigma=\min \left\{t: \eta_{t}^{p} \in \mathscr{P}, t>0\right\}
$$

is the first failure time for $\left(\eta_{s}^{p}\right)$ and

$$
\sigma_{r}=\min \left\{t: \eta_{t}^{p} \in \mathcal{M}, t>\sigma\right\}-\sigma
$$

is the length of repair time (which is the time required for returning to the subset $\mathcal{H}$ ). It follows that

$$
\pi^{\prime}=\frac{\mathbf{E} \int_{0}^{\sigma} I\left(\eta_{t}^{p} \in \cdot\right) d t}{\mathbf{E} \sigma}
$$

Let us decompose process $\left(\eta_{s}^{p}\right)$ into a regenerative Markov process $\left(\eta_{s}^{\prime \prime}\right)$ and a r.v. $T^{\prime \prime}$ such that

$$
\mathbf{P}\left(T^{\prime \prime} \leq t \mid\left(\eta_{s}^{\prime \prime}\right)\right)=1-\exp \left(-\int_{0}^{t} A\left(\eta_{u}^{\prime \prime} ; \Im\right) d u\right) .
$$

This can be done due to Theorem 2.7 (see also (2.4)). Thus, $\left(\eta_{s}^{\prime \prime}\right)$ is a version of the zero-delayed process $\left(\eta_{s}^{0}\right)$. Namely, they both have the same generator $\mathbf{A}^{0}$ (see (2.22)) but different (in general) initial distributions. It follows from (2.5) that

$$
\mathbf{E} \int_{0}^{\sigma} I\left(\eta_{t}^{p} \in \cdot\right) d t=\mathbf{E} \int_{0}^{T^{\prime \prime}} I\left(\eta_{t}^{\prime \prime} \in \cdot\right) d t
$$


Now, define a regenerative process $\eta^{\prime}$ using construction (4.11) but taking i.i.d. copies of $\eta^{\prime \prime}$ as $\eta^{i}$ $(i \geq 1)$. Equip process $\eta^{\prime}$ with regeneration times of the first type (see (4.11) where these times are shown explicitly and the construction of regeneration times given prior to Theorem 4.4). Then stationary distribution $p^{\prime}$ of process $\eta^{\prime}$ has the form

$$
p^{\prime}=\frac{\mathbf{E} \int_{0}^{T^{\prime \prime}} I\left(\eta_{t}^{\prime \prime} \in \cdot\right) d t}{\mathbf{E} T^{\prime \prime}} .
$$

By (5.10) through (5.13), we conclude that $p^{\prime}=\pi^{\prime}$. This completes the proof of the theorem.

The construction of process $\left(\eta_{s}^{\prime}\right)$ is quite similar to the construction of $\left(\widetilde{\eta}_{s}\right)$. Let $\widetilde{S}^{\prime}$ be the first regeneration time of process $\eta^{\prime}$ (the analog of $\widetilde{S}^{1}$ for $\widetilde{\eta}$ ). Then, under assumptions of Theorem 5.1 ,

$$
\lambda_{V}(\infty)=\frac{1}{\mathbf{E}\left(\widetilde{S}^{\prime}\right)} \mathbf{E} \int_{0}^{\widetilde{S}^{\prime}} \alpha\left(\eta_{t}^{\prime}\right) d t
$$

Denote by $S_{0}^{\prime \prime}$ the delay time of the version $\eta^{\prime \prime}$ (an analog of $S_{0}^{1}$ for $\eta^{1}$ ). By arguments like those used in deriving (4.21) and (4.22) from (4.20), we obtain from (5.14) that

where

$$
\lambda_{V}(\infty)=\frac{1}{D_{2}}\left(1-\mathbf{E} \exp \left(-\int_{0}^{S} \alpha\left(\eta_{t}^{0}\right) d t\right)\right),
$$

$$
\begin{gathered}
D_{2}=\mathbf{E} \int_{0}^{S} \exp \left(-\int_{0}^{t} \alpha\left(\eta_{u}^{0}\right) d u\right) d t \mathbf{E}^{\prime \prime} \exp \left(-\int_{0}^{S_{0}^{\prime \prime}} \alpha\left(\eta_{t}^{\prime \prime}\right) d t\right) \\
+\left(1-\mathbf{E} \exp \left(-\int_{0}^{S} \alpha\left(\eta_{t}^{0}\right) d t\right)\right) \mathbf{E}^{\prime \prime} \int_{0}^{S_{0}^{\prime \prime}} \exp \left(-\int_{0}^{t} \alpha\left(\eta_{u}^{\prime \prime}\right) d u\right) d t
\end{gathered}
$$

and $S_{0}^{\prime \prime}$ is the delay time of the version $\eta^{\prime \prime}$ of process $\eta^{0}$ constructed in the proof of Theorem 5.1 (an analog of $S_{0}^{1}$ for $\eta^{1}$ ).

Now, let us use formulas $(4.12),(4.22),(5.15)$ and (5.16) to obtain an estimate of $\rho_{V}$. For this, we are in need of the following conditions.

\section{Conditions C2a}

1. Process $\left(\eta_{s}\right)$ is an ergodic Feller process satisfying the assumptions of Theorem 2.7.

2. Conditions C0 hold true.

Conditions C2b Both $\left(\eta_{s}\right)$ and $\left(\eta_{s}^{0}\right)$ are irreducible Markov processes on a finite state space.

If $\left(\eta_{s}\right)$ meets Condition C2a.1, then, by Theorem 2.7, $\left(\left(\eta_{s}\right), \sigma\right)$ can be decomposed into $\left(\left(\eta_{s}^{0}\right),\left(\alpha_{s}\right)\right)$, with

$$
\alpha_{s}=A\left(\eta_{s}^{0}, \mathscr{\Phi}\right) \equiv \alpha\left(\eta_{s}^{0}\right) .
$$

Condition C2a.2 yields the possibility to use relations (4.21) and (5.15) for estimation of $\rho_{V}$.

If $\left(\eta_{s}\right)$ is a Markov process on a finite state space, then $\left(\eta_{s}^{0}\right)$ is a similar process. However, the irreducibility of $\left(\eta_{s}\right)$ does not yield the irreducibility of $\left(\eta_{s}^{0}\right)$ and vice versa. This is the reason to impose Condition $\mathrm{C} 2 \mathrm{~b}$ in order to use then Remarks 3.5 and 4.5 for estimating $\rho_{V}$ without 
referring to Condition C0.4.

Suppose that either Conditions C2a or C2b are satisfied. By (4.21) and (5.15),

$$
\rho_{V}=\frac{\left|D_{2}-D_{1}\right|}{D_{2}}
$$

Denote

$$
\begin{aligned}
& \beta \equiv \frac{\beta_{N}}{\beta_{D}}=\frac{1-\mathbf{E} \exp \left(-\int_{0}^{S} \alpha\left(\eta_{t}^{0}\right) d t\right)}{\mathbf{E} \int_{0}^{S} \exp \left(-\int_{0}^{t} \alpha\left(\eta_{u}^{0}\right) d u\right) d t} \\
& \xi^{1}=\int_{0}^{S_{0}^{1}} \alpha\left(\eta_{u}^{1}\right) d u, \quad \xi^{\prime \prime}=\int_{0}^{S_{0}^{\prime \prime}} \alpha\left(\eta_{u}^{\prime \prime}\right) d u .
\end{aligned}
$$

We have from (4.22) and (5.16)

Similarly,

$$
\frac{\left|D_{2}-D_{1}\right|}{\beta_{D}} \leq \max \left(\mathbf{E} \xi^{1}, \mathbf{E} \xi^{\prime \prime}\right)+\beta \max \left(\mathbf{E} S_{0}^{1}, \mathbf{E} S_{0}^{\prime \prime}\right)
$$

This yields

$$
\frac{D_{2}}{\beta_{D}} \geq \exp \left(-\mathbf{E} \xi^{\prime \prime}\right)
$$

$$
\rho_{V} \leq \exp \left(\mathbf{E} \xi^{\prime \prime}\right)\left(\max \left(\mathbf{E} \xi^{1}, \mathbf{E} \xi^{\prime \prime}\right)+\beta \max \left(\mathbf{E} S_{0}^{1}, \mathbf{E} S_{0}^{\prime \prime}\right)\right) .
$$

Note that in "typical" reliability problems, $\beta_{D}$ is close to $\mathbf{E} S$ if the system is highly reliable. Using the fact that $\beta_{N} \leq \mathbf{E} \xi$ (where $\xi=\int_{0}^{S} \alpha\left(\eta_{u}^{0}\right) d u$ is defined in (5.4)), we arrive at the bound

$$
\beta \leq \frac{\mathbf{E} \xi}{\mathbf{E} \int_{0}^{S} \exp \left(-\int_{0}^{t} \alpha\left(\eta_{u}^{0}\right) d u\right) d t}
$$

Using (4.21) and (4.22) (resp. (5.15) and (5.16)), we have similarly

and

$$
\lambda(\infty) \leq \frac{1-\mathbf{E} \exp \left(-\int_{0}^{S} \alpha\left(\eta_{t}^{0}\right) d t\right)}{\beta_{D} \mathbf{E} \exp \left(-\int_{0}^{S_{0}^{1}} \alpha\left(\eta_{t}^{1}\right) d t\right)} \leq \frac{1}{\beta_{D}} \exp \left(\mathbf{E} \xi^{1}\right)(1-\exp (-\mathbf{E} \xi))
$$

$$
\lambda_{V}(\infty) \leq \frac{1}{\beta_{D}} \exp \left(\mathbf{E} \xi^{\prime \prime}\right)(1-\exp (-\mathbf{E} \xi))
$$

Let us collect the estimates above as a proposition.

Proposition 5.2: Under Conditions $C 2 a$ or $C 2 b$,

$$
\frac{\left|\lambda_{V}(\infty)-\lambda(\infty)\right|}{\lambda(\infty)} \leq \exp \left(\mathbf{E} \xi^{\prime \prime}\right)\left(\max \left(\mathbf{E} \xi^{1}, \mathbf{E} \xi^{\prime \prime}\right)+\frac{\max \left(\mathbf{E} S_{0}^{1}, \mathbf{E} S_{0}^{\prime \prime}\right) \mathbf{E} \xi}{\mathbf{E} \int_{0}^{S} \exp \left(-\int_{0}^{t} \alpha\left(\eta_{u}^{0}\right) d u\right) d t}\right)
$$

where $S$ is the first regeneration time of $\eta^{0}$,

$$
\xi=\int_{0}^{S} \alpha\left(\eta_{u}^{0}\right) d u, \quad \xi^{1}=\int_{0}^{S_{0}^{1}} \alpha\left(\eta_{u}^{1}\right) d u, \quad \xi^{\prime \prime}=\int_{0}^{S_{0}^{\prime \prime}} \alpha\left(\eta_{u}^{\prime \prime}\right) d u
$$


processes $\eta^{1}$ and $\eta^{\prime \prime}$ are versions of process $\eta^{0}$, and $S_{0}^{1}$ and $S_{0}^{\prime \prime}$ are their delay times. Moreover,

$$
\begin{gathered}
\lambda(\infty) \leq \frac{1}{\beta_{D}} \exp \left(\mathbf{E} \xi^{1}\right)(1-\exp (-\mathbf{E} \xi)), \\
\lambda_{V}(\infty) \leq \frac{1}{\beta_{D}} \exp \left(\mathbf{E} \xi^{\prime \prime}\right)(1-\exp (-\mathbf{E} \xi)) .
\end{gathered}
$$

It is remarkable that estimate (5.18) is expressed in terms of mean characteristics of processes $\eta^{0}, \eta^{1}$, and $\eta^{\prime \prime}$. Because of this, it can be estimated rather easily for a wide class of processes, either analytically or by simulation.

Remark 5.3: Since $S$ is assumed to be $\mathscr{F}_{\infty}^{0}$-measurable, term $\beta_{D}$ in (5.17) can be expressed, with the help of (2.3), as the following mean characteristic:

$$
\left.\beta_{D}=\mathbf{E} \int_{0}^{S} \exp \left(-\int_{0}^{t} \alpha\left(\eta_{u}^{0}\right) d u\right) d t=\mathbf{E} \int_{0}^{S} \mathbf{P}\left(T>t \mid \mathscr{F}_{\infty}^{0}\right)\right) d t=\mathbf{E}(T \wedge S)
$$

\subsection{Markov process with discrete state space}

The bounds derived above can be simplified if one deals with particular random processes. Let us show how they can be written for Markov processes with finite state space.

Let process $\left(\eta_{s}\right)$ be Markov with a finite state space and $A$ be a finite matrix of its transition rates (generator). Assume that $\eta_{0}=0 \in \mathcal{N}$ (in reliability, state 0 is often regarded as the "perfect" operating state). Then, by Theorem 2.7, process $\left(\eta_{s}^{0}\right)$ is Markov evolving on $\mathcal{N}$ with initial state 0 and matrix of transition rates

$$
A^{0}(i, j)=A(i, j) \quad i, j \in \mathcal{M}
$$

Let us view successive entering times to state 0 as regeneration times. We know that processes $\left(\eta_{s}^{1}\right)$ and $\left(\eta_{s}^{\prime \prime}\right)$ are both Markov with the same generator $A^{0}$ but with different initial distributions. In order to keep simple notations, we denote by $\left(\eta_{s}^{0}\right)$ any generic Markov process having generator $\mathbf{A}^{0}$ and by $\mathbf{E}_{\eta}$ the conditional expectation given $\eta_{0}^{0}=\eta$. Let

$$
\tau_{0}=\inf \left\{t: \eta_{t-}^{0} \neq \eta_{t}^{0}, \eta_{t}^{0}=0\right\}
$$

be the first returning time of process $\left(\eta_{s}^{0}\right)$ to state 0 . Then

$$
\max \left(\mathbf{E} \xi^{1}, \mathbf{E} \xi^{\prime \prime}\right) \leq \sup _{\eta \neq 0} \mathbf{E}_{\eta} \int_{0}^{\tau_{0}} \alpha\left(\eta_{u}^{0}\right) d u \text { and } \max \left(\mathbf{E} S_{0}^{1}, \mathbf{E} S_{0}^{\prime \prime}\right) \leq \sup _{\eta \neq 0} \mathbf{E}_{\eta} \tau_{0}
$$

Since $S$ is the first returning time of $\eta^{0}$ to the state 0 , by similar arguments as those in Remarks 5.3 and $(2.2)$, we arrive at the equality

$$
\beta_{D}=\mathbf{E}_{0}\left(\sigma \wedge \sigma_{0}\right)
$$

where $\sigma_{0}$ is the first returning time of $\left(\eta_{s}\right)$ to the state 0 .

Let us note that the right-hand side of (5.18) is also an upper bound for $\mid \lambda_{V}(\infty)-$ $\lambda(\infty) \mid / \lambda_{V}(\infty)$. Bringing this together with the above estimates and Proposition 5.2, we arrive at the following corollary.

Corollary 5.4: Given Condition $C 2 b$, let $\epsilon, \epsilon_{0}, \delta, \underline{\delta}_{0}$, and $\underline{\beta}_{D}$ be such that

$$
\sup _{\eta \neq 0} \mathbf{E} \int_{0}^{\tau_{0}} \alpha\left(\eta_{u}^{0}\right) d u \leq \epsilon, \quad \mathbf{E}_{0} \int_{0}^{\tau_{0}} \alpha\left(\eta_{u}^{0}\right) d u \leq \epsilon_{0}, \sup _{\eta \neq 0} \mathbf{E}_{\eta} \tau_{0} \leq \delta, \quad \underline{\delta}_{0} \leq \mathbf{E}_{0} \tau_{0}, \quad \underline{\beta}_{D} \leq \mathbf{E}_{0}\left(\sigma \wedge \sigma_{0}\right) .
$$


Then

$$
\frac{\lambda_{V}(\infty)}{1+\rho_{V}} \leq \lambda(\infty) \leq\left(1+\rho_{V}\right) \lambda_{V}(\infty)
$$

with

$$
\rho_{V} \leq e^{\epsilon}\left(\epsilon+\frac{\delta \epsilon_{0}}{\underline{\beta}_{D}}\right)
$$

Moreover,

$$
\max \left(\lambda(\infty), \lambda_{V}(\infty)\right) \leq \frac{1}{\underline{\beta}_{D}} e^{\epsilon}\left(1-e^{-\epsilon_{0}}\right), \quad \lambda_{0} \leq \frac{\epsilon_{0}}{\underline{\delta}_{0}} .
$$

In [6], we will show how to calculate parameters $\epsilon, \epsilon_{0}, \delta, \underline{\delta}_{0}$ and $\underline{\beta}_{D}$. Sometimes it can be done explicitly, but generally they have to be estimated. These estimates may be more or less accurate. The following crude estimates are evident:

$$
\begin{gathered}
\epsilon=\max _{\eta \neq 0} \alpha(\eta), \\
\epsilon_{0}=\frac{\alpha(0)}{\left|A^{0}(0,0)\right|}+\epsilon \\
\underline{\delta}_{0}=\frac{1}{\left|A^{0}(0,0)\right|}+\sum_{\eta \neq 0} \frac{A^{0}(0, \eta)}{A^{0}(0,0) A^{0}(\eta, \eta)} \\
\underline{\beta}_{D}=\frac{1}{|A(0,0)|}+\sum_{\eta \in \mathcal{M}, \eta \neq 0} \frac{A(0, \eta)}{A(0,0) A(\eta, \eta)} .
\end{gathered}
$$

Value $\delta$ can be estimated with the help of test functions and the following result (see Kalashnikov [15] (Chapter 1, Corollary 1)).

Lemma 5.5: Let $V$ be a non-negative function such that

$$
\mathbf{A}^{0} V(\eta) \leq-1, \quad \forall \eta \neq 0 .
$$

Then,

$$
\mathbf{E}_{\eta} \tau_{0} \leq V(\eta), \quad \forall \eta \neq 0
$$

and one can take

$$
\delta=\sup _{\eta \neq 0} V(\eta)
$$

\section{Acknowledgements}

A considerable part of this work was done during V. Kalashnikov's visit at the Department of Analysis and Applied Mathematics of the University of Marne-La-Vallée. The visitor is thankful to his co-author and Michel Roussignol for the friendly atmosphere and fruitful discussions.

\section{References}

[1] Asmussen, S., Applied Probability and Queues, J. Wiley \& Sons, New York 1992.

[2] Çinlar, E., Introduction to Stochastic Processes, Prentice-Hall, Englewood Cliffs, New Jersey 1975.

[3] Cocozza-Thivent, C. and Roussignol, M., Quasi-stationary probability for a $r$-positive semi-Markov process and applications to reliability, Prépublications de l'Equipe d'Analyse 
et de Mathématiques Appliquées, Université de Marne-la-Vallée, 11, October 1994.

[4] Cocozza-Thivent, C. and Roussignol, M., Comparaison des lois stationnaire et quasistationnaire d'un processus de Markov et applications à la fiabilité, Seminaire de Probabilités XXX, Lecture Notes in Mathematics 1626, Springer-Verlag (1996), 24-39.

[5] Cocozza-Thivent, C. and Roussignol, M., Techniques de couplage en fiabilité, Ann. I.H.P. 31:1 (1995), 119-141.

[6] Cocozza-Thivent, C. and Kalashnikov, V., The failure rate in reliability: Numerical treatment, J. Appl. Math. Stoch. Anal., to appear.

[7] Davis, M.H.A., Piecewise-deterministic Markov processes: A general class of non-diffusion models, J. Roy. Stat. Soc. B 46 (1984), 353-388.

[8] Disney, R. and Kiessler, P., Traffic Processes in Queueing Networks. A Markov Renewal Approach, John Hopkins Univ. Press, Baltimore 1987

[9] Dynkin, E., Markov Processes, Vol. I, II, Springer-Verlag, Berlin 1965.

[10] Ethier, S.N. and Kurtz, T.G., Markov Processes: Characterization and Convergence, J. Wiley \& Sons, New York 1986.

[11] Feller, W., An Introduction to Probability Theory and Its Applications, Vol. 2, J. Wiley \& Sons, New York 1986.

[12] Franken, P., König, D., Arndt, U., and Schmit, V., Queues and Point Processes, Akademie-Verlag, Berlin 1981.

[13] Jacod, J. and Shiryaev, A.N., Limit Theorems for Stochastic Processes, Springer-Verlag, Berlin 1987.

[14] Kalashnikov, V., A Qualitative Analysis of Complex Systems by the Test Functions Method, Nauka, Moscow 1978. [Russian]

[15] Kalashnikov, V., Mathematical Methods in Queueing Theory, Kluwer Acad. Publ., Dordrecht 1994.

[16] Kalashnikov, V., Topics on Regenerative Processes, CRC Press, Boca Raton 1994.

[17] Kalashnikov, V., Quantitative estimates in queueing, In: Advances in Queueing, Methods and Open Problems (ed. by J. Dshalalow), CRC Press, Boca Raton (1995), 407-428.

[18] Lindvall, T., Lectures on the Coupling Method, J. Wiley \& Sons, New York 1992.

[19] Meyn, S. and Tweedie, R., Markov Chains and Stochastic Stability, Springer-Verlag, New York 1993.

[20] Pagès, A. and Gondran, M., Fiabilité des Systèmes, Eyrolles, Collection de la Direction des Etudes et Recherches d'Electricité de France, Paris 1980.

[21] Shaked, M. and Shanthikumar, G.J., Stochastic Orders and Their Applications, Academic Press, San Diego 1994.

[22] Thorisson, H., Construction of a stationary regenerative process, Stoch. Proc. Appl. 42 (1992), 237-253. 


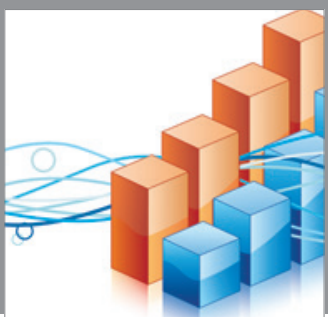

Advances in

Operations Research

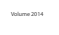

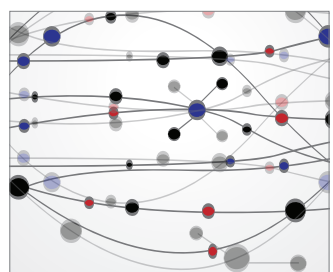

\section{The Scientific} World Journal
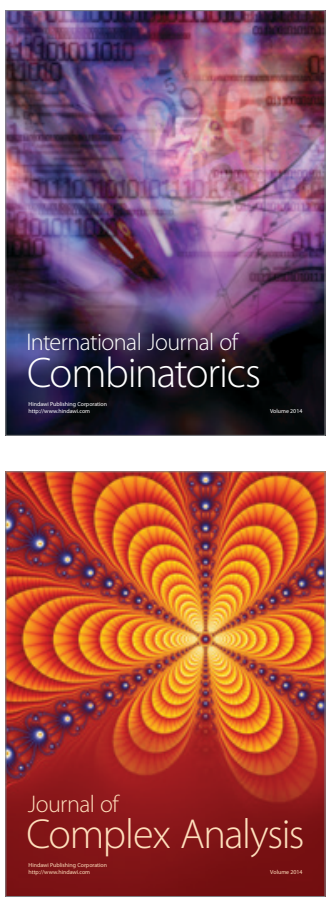

International Journal of

Mathematics and

Mathematical

Sciences
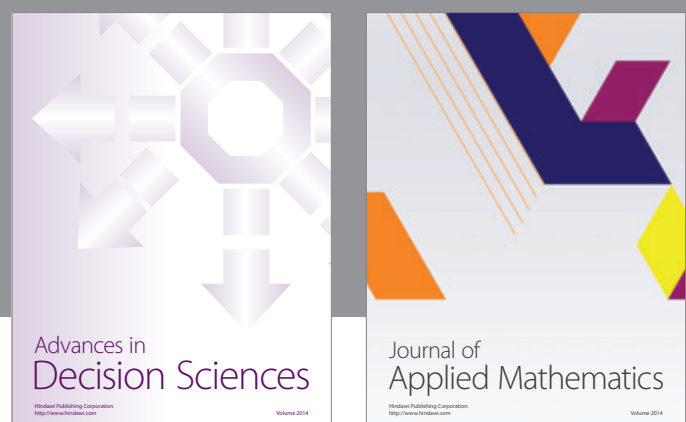

Journal of

Applied Mathematics
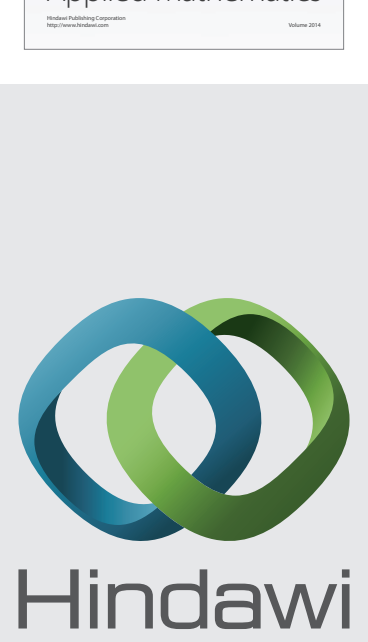

Submit your manuscripts at http://www.hindawi.com
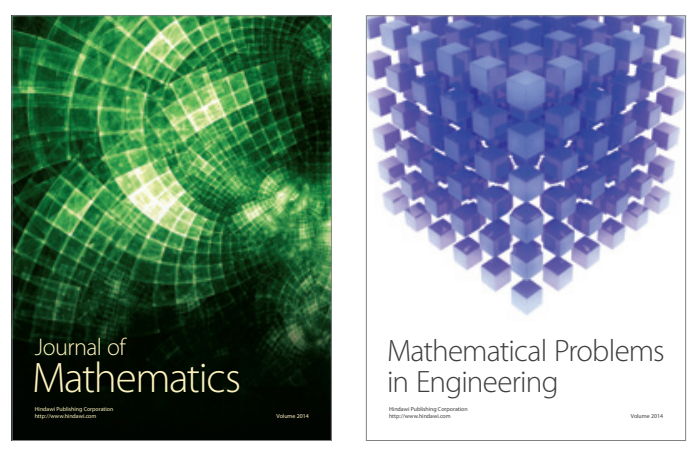

Mathematical Problems in Engineering
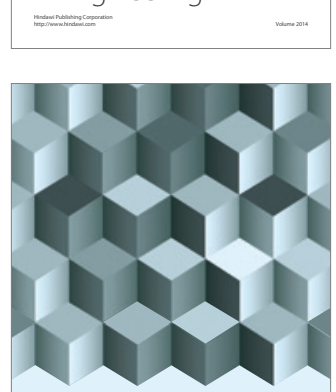

Journal of

Function Spaces
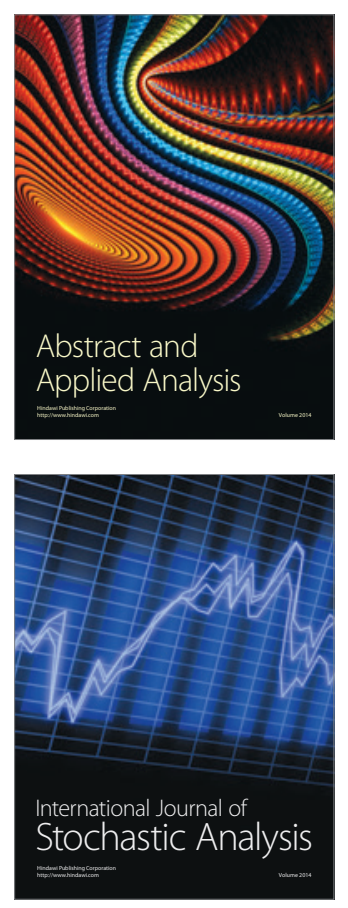

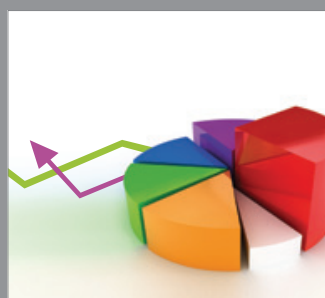

ournal of

Probability and Statistics

Promensencen
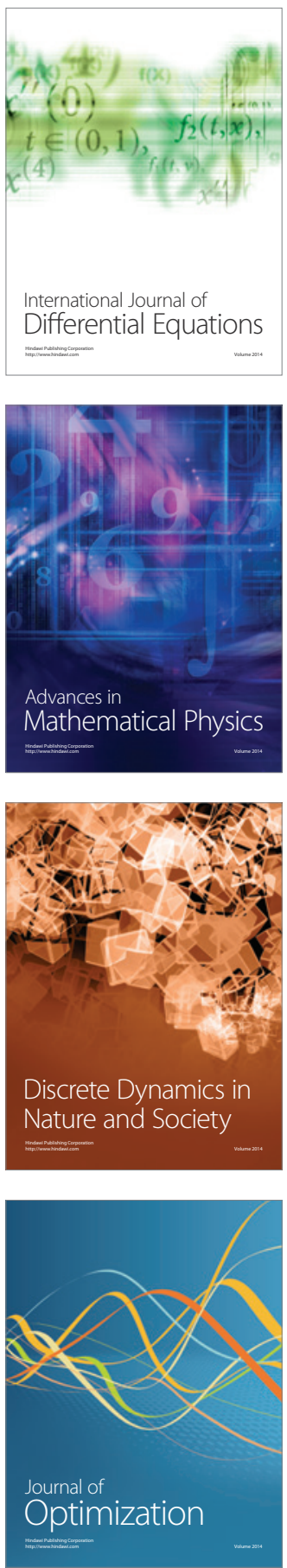